\title{
Seislet transform and seislet frame ${ }^{a}$
}

${ }^{a}$ Published in Geophysics, 75, no. 3, V25-V38, (2010)

Sergey Fomel and Yang Liu

\begin{abstract}
We introduce a digital wavelet-like transform, which is tailored specifically for representing seismic data. The transform provides a multiscale orthogonal basis with basis functions aligned along seismic events in the input data. It is defined with the help of the wavelet lifting scheme combined with local planewave destruction. In the $1-\mathrm{D}$ case, the seislet transform is designed to follow locally sinusoidal components. In the $2-\mathrm{D}$ case, it is designed to follow local plane wave components with smoothly variable slopes. If more than one component is present, the transform turns into an overcomplete representation or a tight frame. In these terms, the classic digital wavelet transform is simply a seislet transform for a zero frequency (in 1-D) or zero slope (in 2-D).

The main objective of the new transform is an effective seismic data compression for designing efficient data analysis algorithms. Traditional signal processing tasks such as noise attenuation and trace interpolation become simply defined in the seislet domain. When applied in the offset direction on common midpoint or common image point gathers, the seislet transform finds an additional application in optimal stacking of seismic records.
\end{abstract}

\section{INTRODUCTION}

Wavelet transforms have found many applications in science and engineering (Mallat, 2009), including geophysics (Foster et al., 1994; Dessing, 1997; Wapenaar et al., 2005; Kazemeini et al., 2009). The power of wavelet transforms, in comparison with the classic Fourier transform, lies in their ability to represent non-stationary signals. As a result, wavelets can provide a compact basis for non-stationary data decomposition. Having a compact basis is useful both for data compression and for designing efficient numerical algorithms.

A number of wavelet-like transforms that explore directional characteristics of images have been proposed in the image analysis literature (Welland, 2003). Among those transforms are bandelets (Pennec and Mallat, 2005), contourlets (Do and Vetterli, 2005), curvelets (Starck et al., 2002), directionlets (Velisavljevic, 2005), shearlets (Guo and Labate, 2007), etc. Unlike isotropic wavelets, directional transforms attempt to design basis functions so that they appear elongated anisotropically along 2-D curves or 3-D surfaces, which might be characteristic for an image. Therefore, 
these transforms achieve better accuracy and better data compression in representing non-stationary images with curved edges. Curvelets are particularly appropriate for seismic data because they provide a provably optimal decomposition of wavepropagation operators (Candés and Demanet, 2005). Application of the curvelet transform to seismic imaging and seismic data analysis has been an area of active research (Herrmann et al., 2007; Douma and de Hoop, 2007; Chauris and Nguyen, 2008; Herrmann and Hennenfent, 2008).

Although the wavelet theory originated in seismic data analysis (Morlet, 1981), none of the known wavelet-like transforms were designed specifically for seismic data. Even though some of the transforms are applicable for representing seismic data, their original design was motivated by different kinds of data, such as piecewise-smooth images. In this paper, we investigate the possibility of designing a transform tailored specifically for seismic data. In analogy with the previous naming games, we call such a transform the seislet transform (Fomel, 2006).

In seismic data analysis, it is common to represent signals as sums of sinusoids (in 1-D) or plane waves (in 2-D) with the help of the digital Fourier transform (DFT). Certain methods for seismic data regularization, such as the anti-leakage Fourier transform (Xu et al., 2005), the Fourier reconstruction method (Zwartjes and Gisolf, 2006, 2007; Zwartjes and Sacchi, 2007), or POCS (Abma and Kabir, 2006) rely on the ability to represent signals sparsely in the transform domain. The digital wavelet transform (DWT) is often preferred to the Fourier transform for characterizing digital images, because of its ability to localize events in both time and frequency domains (Jensen and la Cour-Harbo, 2001; Mallat, 2009). However, DWT may not be optimal for describing data that consist of individual sinusoids or plane waves. It is for those kinds of data that the seislet transform attempts to achieve an optimally compact representation.

The approach taken in this paper follows the general recipe for digital wavelet transform construction known as the lifting scheme (Sweldens, 1995). The lifting scheme provides a convenient and efficient construction for digital wavelet transforms of different kinds. The key ingredients of this scheme are a prediction operator and an update operator defined at different digital scales. The goal of the prediction operator is to predict regular parts of the input data so that they could be subtracted from the analysis. The goal of the update operator is to carry essential parts of the input data to the next analysis scale. Conventional wavelet transforms use prediction and update operators designed for characterizing locally smooth images. In this paper, we show how designing prediction and update tailored for seismic data can improve the effectiveness of the transform in seismic applications. In 1-D, our prediction and update operators focus on predicting sinusoidal signals with chosen frequencies. In 2-D and 3-D, we use predictions along locally dominant event slopes found by the method of plane-wave destruction (Claerbout, 1992; Fomel, 2002). One can extend the idea of the seislet transform further by changing the definition of prediction and update operators in the lifting scheme (Liu and Fomel, 2009).

The seislet transform decomposes a seismic image into an orthogonal basis which 
is analogous to the wavelet basis but aligned along dominant seismic events. In 1$\mathrm{D}$, the classic wavelet transform is equivalent to the seislet transform with a zero frequency. In 2-D, the wavelet transform in the horizontal direction is equivalent to the seislet transform with a zero slope. When more than one frequency or more than one slope field are employed for analysis, the seislet transform turns into an overcomplete representation or a tight frame.

The paper is organized as follows. We start by reviewing the digital wavelet transform and the lifting scheme. Next, we modify the lifting scheme to define 1-D and 2-D seislet transforms. Finally, we generalize the transform construction to a frame. We illustrate applications of both the seislet transform and the seislet frame with synthetic and field data examples.

\section{LIFTING SCHEME FOR WAVELET TRANSFORMS}

In order to define the new transform, we follow the general recipe for digital wavelet transforms provided by Sweldens and Schröder (1996). In the most general terms, the lifting scheme (Sweldens, 1995) can be defined as follows:

1. Organize the input data as a sequence of records.

2. Break the data into even and odd components e and o.

3. Find a residual difference $\mathbf{r}$ between the odd component and its prediction from the even component:

$$
\mathbf{r}=\mathbf{o}-\mathbf{P}[\mathbf{e}]
$$

where $\mathbf{P}$ is a prediction operator.

4. Find a coarse approximation $\mathbf{c}$ of the data by updating the even component

$$
\mathbf{c}=\mathbf{e}+\mathbf{U}[\mathbf{r}]
$$

where $\mathbf{U}$ is an update operator.

5. The coarse approximation $\mathbf{c}$ becomes the new data, and the sequence of steps is repeated at the next scale level.

A digital wavelet transform consists of data approximation at the coarsest level and residuals from all the levels. The key in designing an effective transform is making sure that the prediction operator $\mathbf{P}$ leaves small residuals while the update operator $\mathbf{U}$ preserves essential features of the original data while promoting them to the next scale level. For example, one can obtain the classic Haar wavelet by defining the prediction operator as a simple shift from the nearest sample:

$$
\mathbf{P}[\mathbf{e}]_{k}=\mathbf{e}_{k}
$$


with the update operator designed to preserve the DC (zero frequency) component of the signal. Alternatively, the $(2,2)$ Cohen-Daubechies-Feauveau biorthogonal wavelets (Cohen et al., 1992) are constructed by making the prediction operator a linear interpolation between two neighboring samples,

$$
\mathbf{P}[\mathbf{e}]_{k}=\left(\mathbf{e}_{k-1}+\mathbf{e}_{k}\right) / 2,
$$

and by constructing the update operator to preserve the running average of the signal (Sweldens and Schröder, 1996), as follows:

$$
\mathbf{U}[\mathbf{r}]_{k}=\left(\mathbf{r}_{k-1}+\mathbf{r}_{k}\right) / 4
$$

The digital wavelet transform is an efficient operation. Assuming that the prediction and update operation take a constant cost per record, the number of operations at the finest scale is proportional to the total number of records $N$, the next scale computation takes $O(N / 2)$, etc. so that the total number of operations is proportional to $N+N / 2+N / 4+\ldots+2=2(N-1)$, which is smaller than the $O(N \log N)$ cost of the Fast Fourier Transform.

The digital wavelet transform is also easily invertible. Reversing the lifting scheme operations provides the inverse transform algorithm, as follows:

1. Start with the coarsest scale data representation $\mathbf{c}$ and the coarsest scale residual r.

2. Reconstruct the even component e by reversing the operation in equation 2 , as follows:

$$
\mathbf{e}=\mathbf{c}-\mathbf{U}[\mathbf{r}]
$$

3. Reconstruct the odd component o by reversing the operation in equation 1 , as follows:

$$
\mathbf{o}=\mathbf{r}+\mathbf{P}[\mathbf{e}]
$$

4. Combine the odd and even components to generate the data at the previous scale level and repeat the sequence of steps.

Figure 1 shows a classic benchmark image from the image analysis literature and its digital wavelet transform using 2-D biorthogonal wavelets. Thanks to the general smoothness of the "Lena" image, the residual differences from equation 2 (stored as wavelet coefficients at different scales) have a small dynamic range, which enables an effective compression of the image in the transform domain. Wavelet compression algorithms are widely used in practice for compression of natural images. As for compression of seismic data, the classic DWT may not be optimal, because it does not take into account the specific nature of seismic data patterns. In the next section, we turn the wavelet transform into the seislet transform, which is tailored for representing seismic data. 


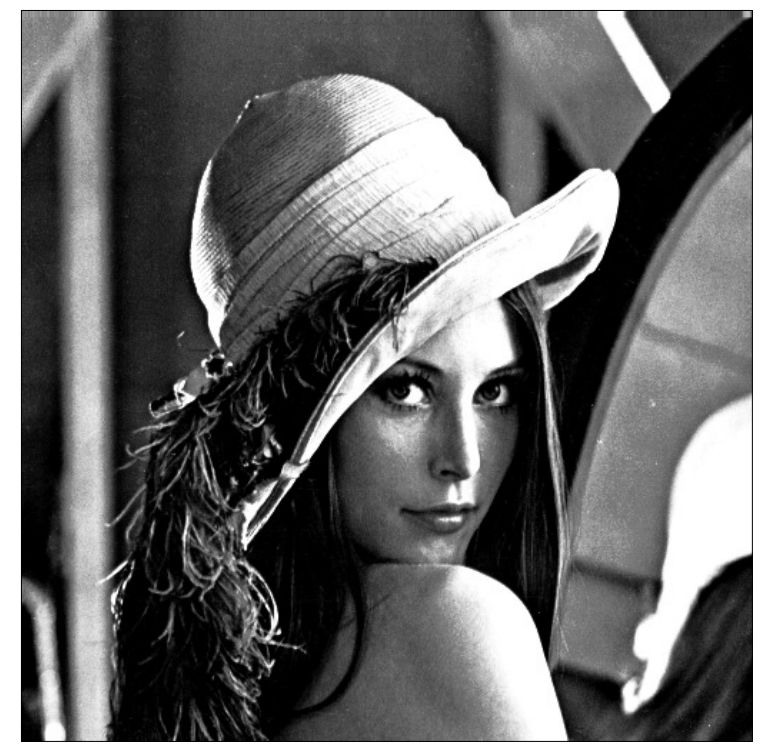

Lena

(a)

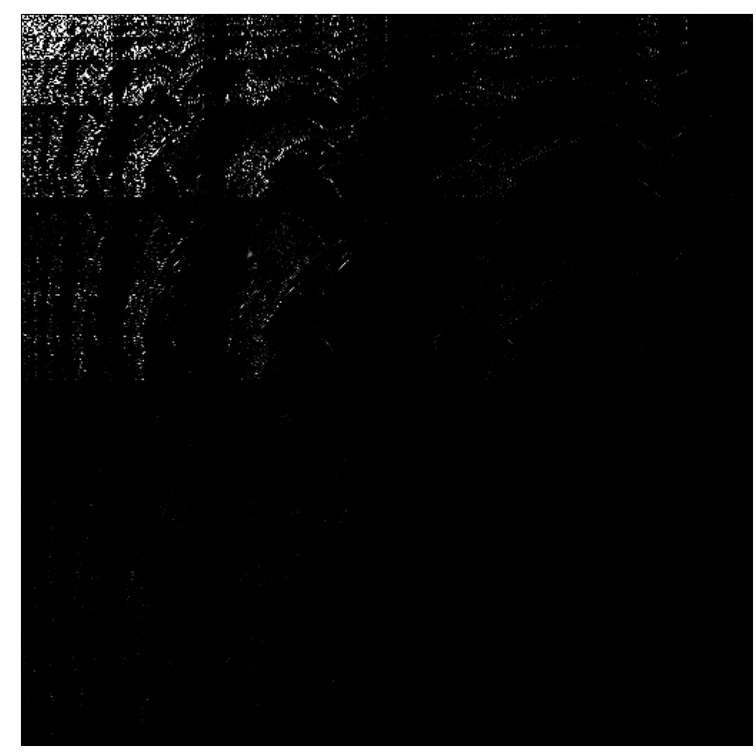

2-D Wavelet Transform

(b)

Figure 1: Benchmark "Lena" image from image analysis literature (a) and its 2-D digital wavelet transform using bi-orthogonal wavelets (b). 


\section{FROM WAVELETS TO SEISLETS}

We adopt the general idea of the lifting scheme to transforming seismic data. The key idea of the seislet transform (Fomel, 2006) is recognizing that

- seismic data can be organized as collections of traces or records;

- prediction of one seismic trace or record from the other and update of records on the next scale should follow features characteristic for seismic data.

\section{1-D seislet transform}

The prediction and update operators employed in the lifting scheme can be understood as digital filters. In the $Z$-transform notation, the Haar prediction filter from equation 3 is

$$
P(Z)=Z
$$

(shifting each sample by one), and the linear interpolation filter from equation 4 is

$$
P(Z)=1 / 2(1 / Z+Z)
$$

These predictions are appropriate for smooth signals but may not be optimal for a sinusoidal signal. In comparison, the prediction

$$
P(Z)=Z / Z_{0}
$$

where $Z_{0}=e^{i \omega_{0} \Delta t}$, perfectly characterizes a sinusoid with $\omega_{0}$ circular frequency sampled on a $\Delta t$ grid. In other words, if a constant signal $\left(\omega_{0}=0\right)$ is perfectly predicted by shifting each trace to its neighbor, a sinusoidal signal $\left(\omega_{0} \neq 0\right)$ requires the shift to be modulated by an appropriate frequency. Likewise, the linear interpolation in equation 9 needs to be replaced by a filter tuned to a particular frequency in order to predict a sinusoidal signal with that frequency perfectly:

$$
P(Z)=1 / 2\left(Z_{0} / Z+Z / Z_{0}\right)
$$

The analysis easily extends to higher-order filters.

\section{2-D seislet transform}

If we view seismic data as collections of traces, we can predict one trace from the other by following local slopes of seismic events. Such a prediction is a key operation in the method of plane-wave destruction (Fomel, 2002). In fact, it is the minimization of prediction error that provides a criterion for estimating local slopes (Claerbout, 1992). For completeness, we include a review of plane-wave destruction in the appendix. 
The prediction and update operators for a simple seislet transform are defined by modifying the biorthogonal wavelet construction in equations 4-5 as follows:

$$
\begin{aligned}
& \mathbf{P}[\mathbf{e}]_{k}=\left(\mathbf{S}_{k}^{(+)}\left[\mathbf{e}_{k-1}\right]+\mathbf{S}_{k}^{(-)}\left[\mathbf{e}_{k}\right]\right) / 2 \\
& \mathbf{U}[\mathbf{r}]_{k}=\left(\mathbf{S}_{k}^{(+)}\left[\mathbf{r}_{k-1}\right]+\mathbf{S}_{k}^{(-)}\left[\mathbf{r}_{k}\right]\right) / 4,
\end{aligned}
$$

where $\mathbf{S}_{k}^{(+)}$and $\mathbf{S}_{k}^{(-)}$are operators that predict a trace from its left and right neighbors correspondingly by shifting seismic events according to their local slopes. The predictions need to operate at different scales, which, in this case, mean different separation distances between the traces. Equations 12-13, in combination with the forward and inverse lifting schemes 1-2 and 6-7, provide a complete definition of the 2-D seislet transform.

Figure 2(a) shows a synthetic seismic image from Claerbout (2008). After estimating local slopes from the image by plane-wave destruction (Figure 2(b)), we applied the 2-D seislet transform described above. The transform is shown in Figure 3(b) and should be compared with the corresponding wavelet transform in Figure 3(a). Apart from the fault and unconformity regions, where the image is not predictable by continuous local slopes, the 2-D seislet transform coefficients are small, which enables an effective compression. In contrast, the wavelet transform has small residual coefficients at fine scales but develops large coefficients at coarser scales. Figure 4 shows a comparison between the decay of coefficients (sorted from large to small) between the wavelet transform and the seislet transform. A significantly faster decay of the seislet coefficients is evident.

Effectively, the wavelet transform in this case is equivalent to the 2-D seislet transform with the erroneous zero slope. Figure 5 shows example basis functions for the wavelet and 2-D seislet transform used in this example. If using only a small number of the most significant coefficients, the wavelet transform fails to reconstruct the most important features of the original image, while the 2-D seislet transform achieves an excellent reconstruction (Figure 6). We use the method of soft thresholding (Donoho, 1995) for selecting the most significant coefficients.

\section{Example applications of 2-D seislet transform}

In this section, we discuss some example applications of the 2-D seislet transform.

\section{Denoising and trace interpolation}

Figure $7(\mathrm{a})$ shows a common-midpoint gather from a North Sea dataset. Plane-wave destruction estimates local slopes shown in Figure 7(b) and enables the 2-D seislet transform shown in Figure 8(a). Small dynamic range of seislet coefficients implies a good compression ratio. Figure 8 (b) shows data reconstruction using only $5 \%$ of the significant seislet coefficients. 


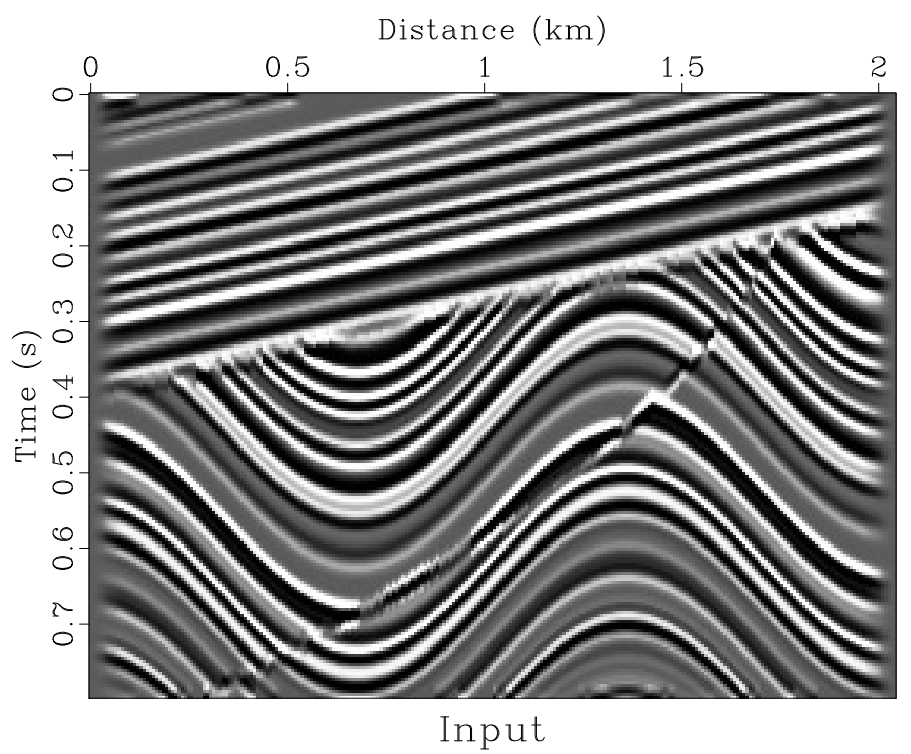

(a)

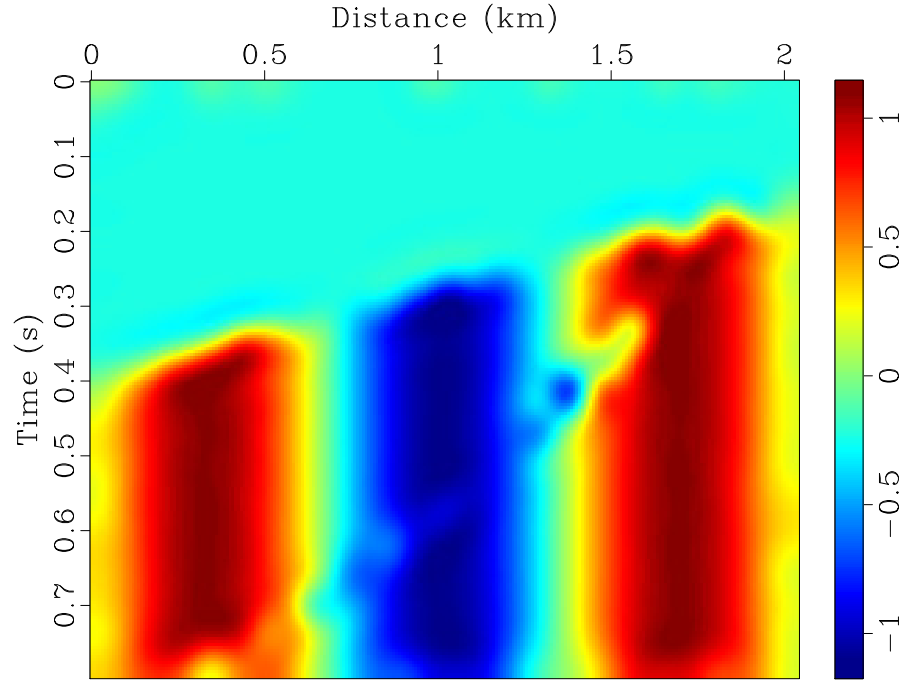

Slope

(b)

Figure 2: Synthetic seismic image (a) and local slopes estimated by plane-wave destruction (b). 


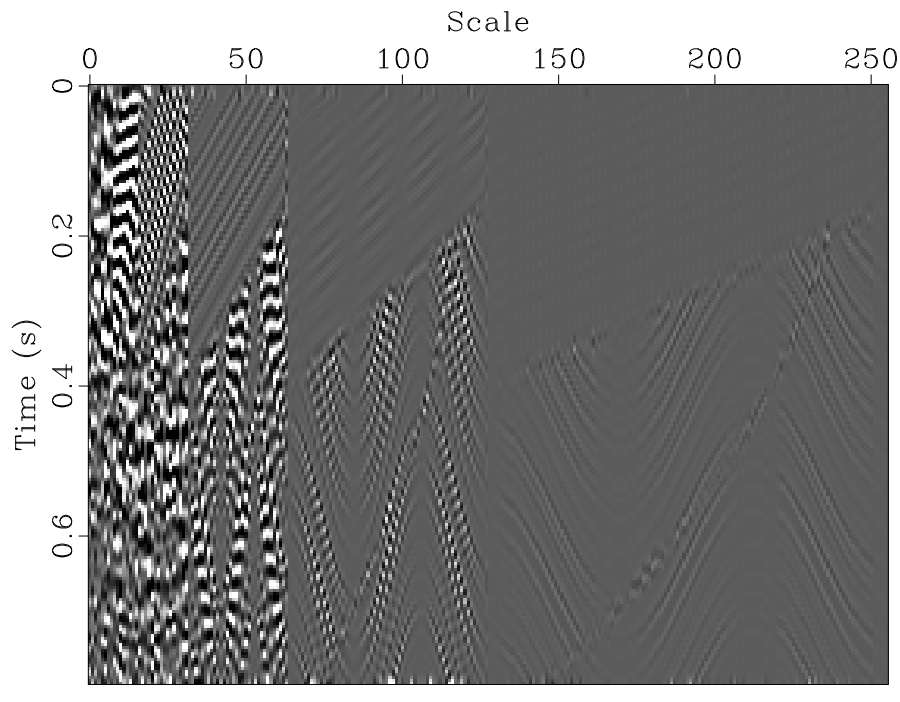

Wavelet Transform

(a)

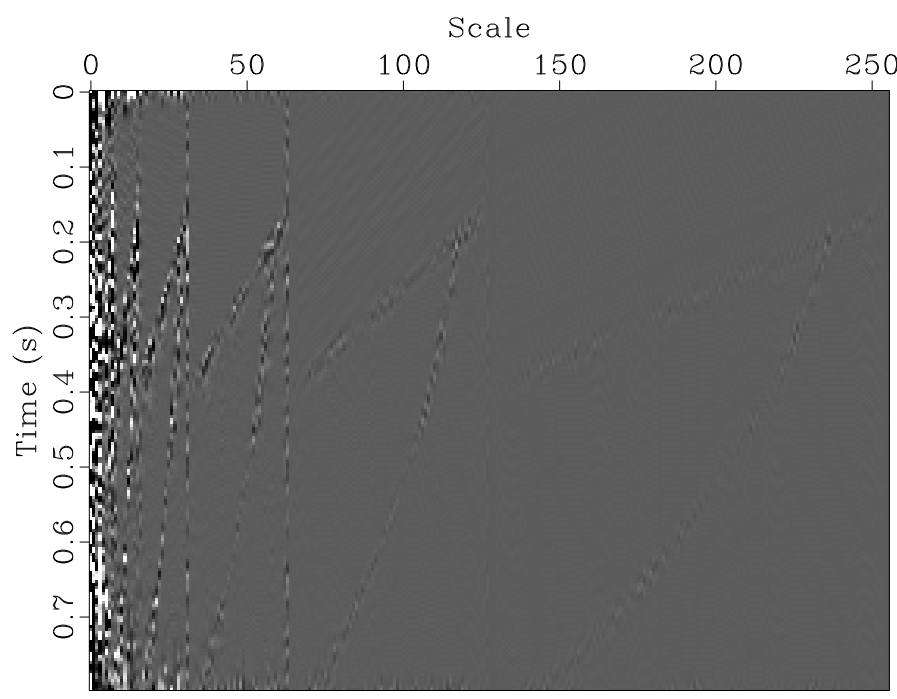

Seislet Transform

(b)

Figure 3: Wavelet transform (a) and seislet transform (b) of the synthetic image from Figure 2. 


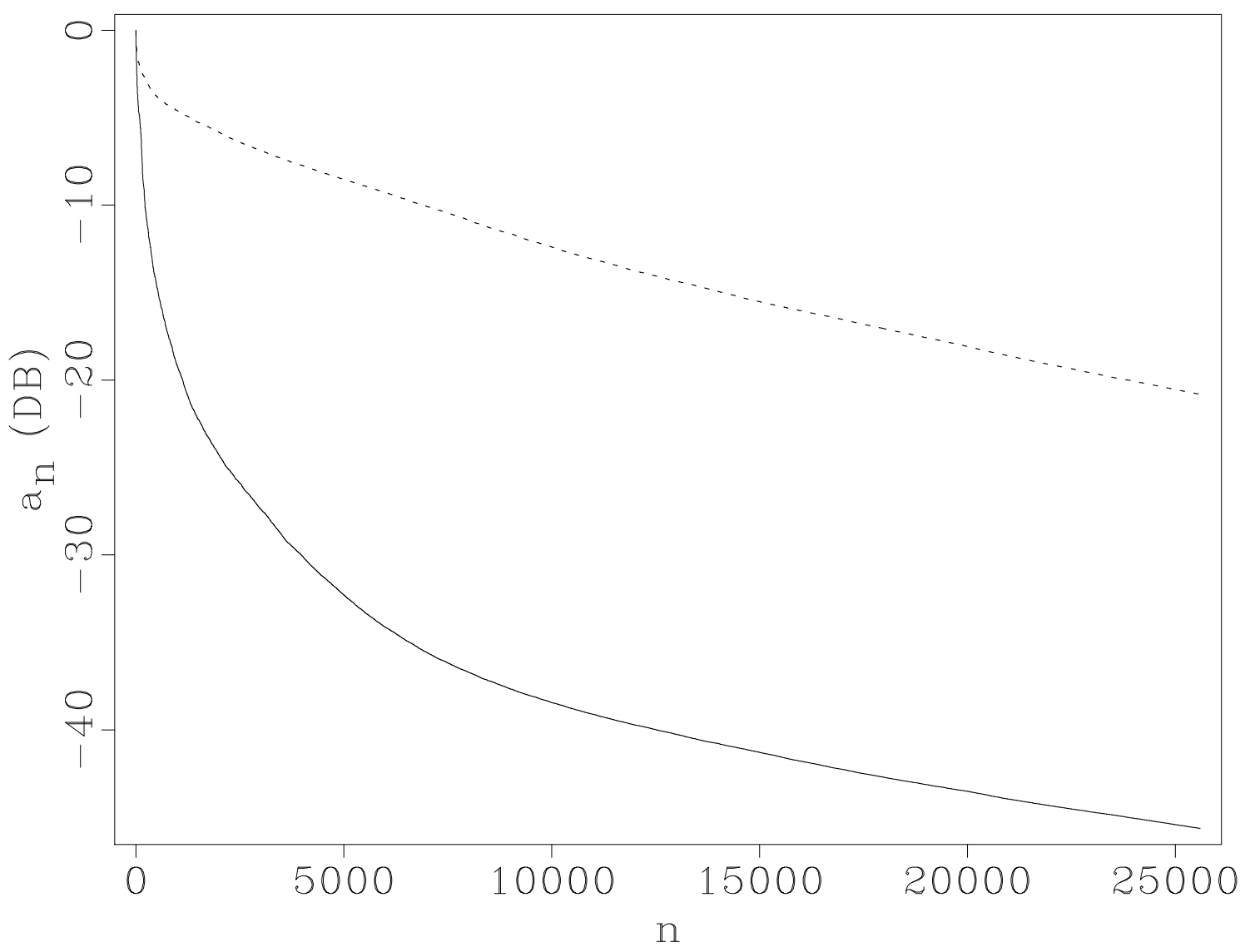

Figure 4: Transform coefficients sorted from large to small, normalized, and plotted on a decibel scale. Solid line: seislet transform. Dashed line: wavelet transform. 


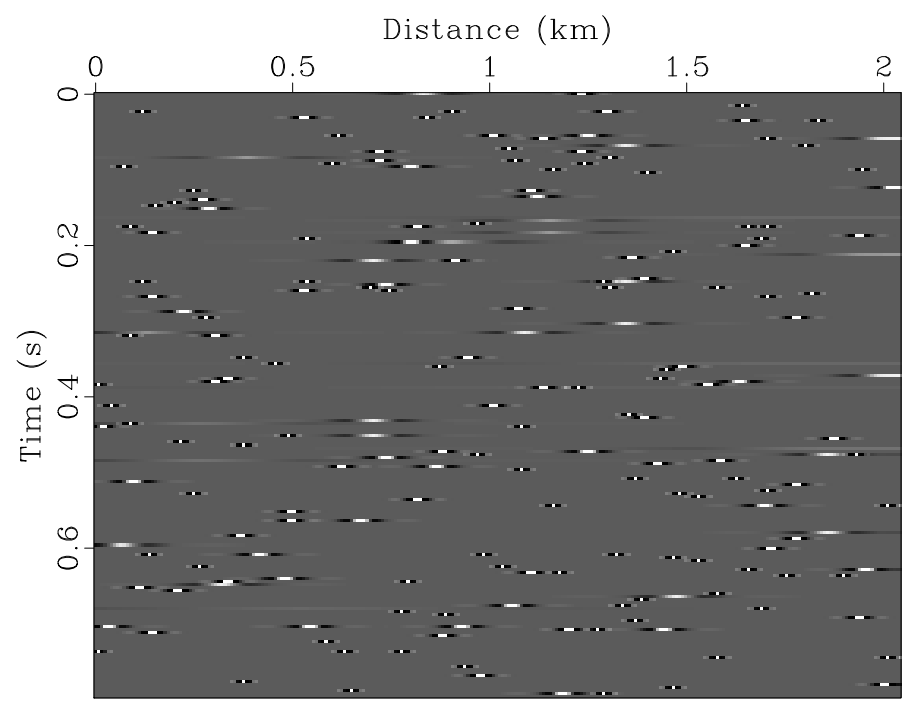

Wavelets

(a)

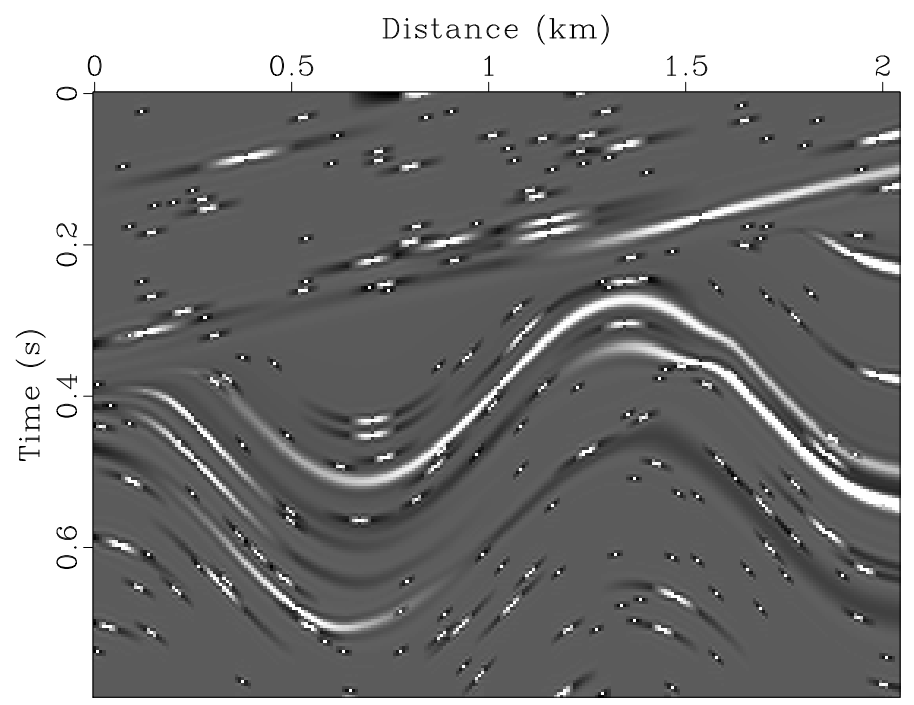

Seislets

(b)

Figure 5: Randomly selected representative basis functions for wavelet transform (a) and seislet transform (b). 


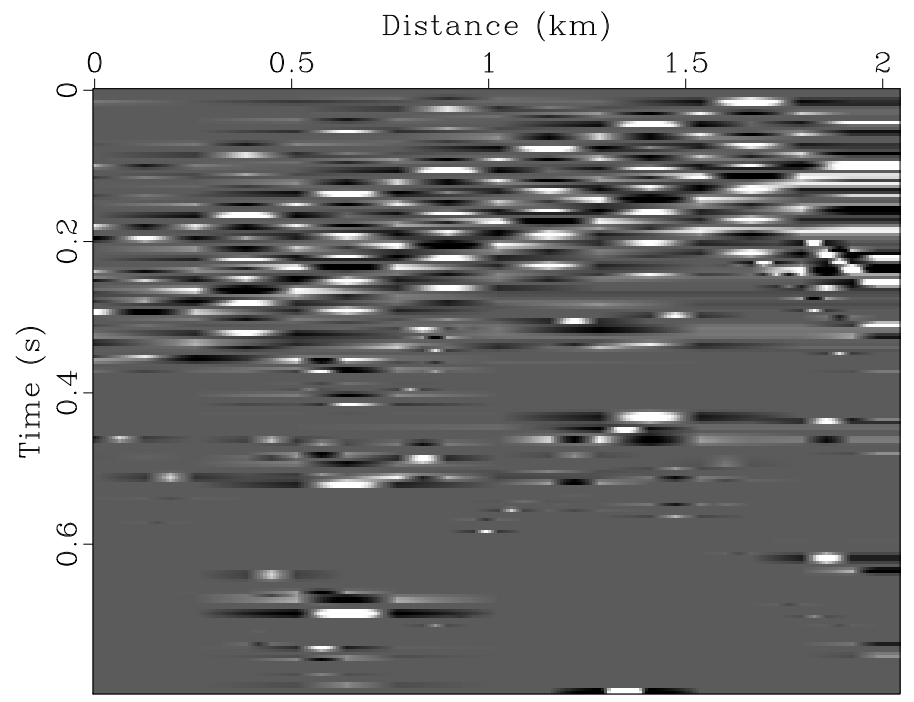

Inverse Wavelet Transform (1\%)

(a)

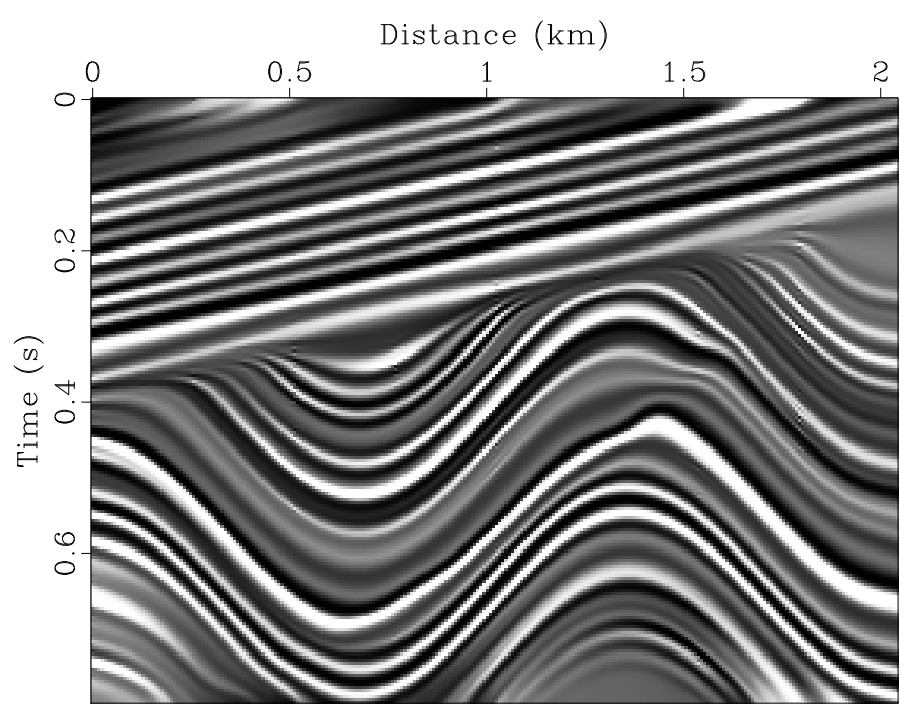

Inverse Seislet Transform (1\%)

(b)

Figure 6: Synthetic image reconstruction using only $1 \%$ of significant coefficients (a) by inverse wavelet transform (b) by inverse seislet transform. Compare with Figure 2(a). 
If we choose the significant coefficients at the coarse scale and zero out difference coefficients at the finer scales, the inverse transform will effectively remove incoherent noise from the gather (Figure 8). Thus, denoising is a naturally defined operation in the 2-D seislet domain (Figure 9).

If we extend the seislet transform domain and interpolate the smooth local slope to a finer grid, the inverse seislet transform will accomplish trace interpolation of the input gather (Figure 10). We extend not simply with zeros but with small random noise to account for the fact that realistic noise is unpredictable and therefore exists on different scale levels. In this example, the number of traces is increased by four. Thus, trace interpolation also turns out to be a natural operation when viewed from the 2-D seislet domain.

\section{Seislet stack}

The seislet transform acquires a special meaning when applied in the offset direction on common midpoint or common image point gathers. According to the lifting construction, the zero-order seislet coefficient is nothing more than seismic stack computed in a recursive manner by successive partial stacking of neighboring traces. As a consequence, seislet stack avoids the problem of "NMO stretch" associated with usual stacking (Haldorsen and Farmer, 1989) as well as the problem of nonhyperbolic moveouts Fomel and Grechka (2001). All other gather attributes including multiple reflections and amplitude variation with offset appear in the higher order seislet coefficients. Figure 11 shows a comparison between the conventional normal moveout stack and the seislet stack. The higher resolution of the seislet stack is clearly visible. Figure 12 compares the common-midpoint gather after conventional normal moveout correction and an effective seislet moveout computed by separating contributions from individual traces to the seislet stack.

\section{FROM TRANSFORM TO FRAME}

The 1-D and 2-D transforms, defined in the previous sections, are appropriate for analyzing signals, which have a single dominant sinusoid or plane-wave component. In practice, it is common to analyze signals composed of multiple sinusoids (in 1-D) or plane waves (in 2-D). If a range of frequencies or plane-wave slopes is chosen, and the appropriate transform is constructed for each of them, all the transform domains taken together will constitute an overcomplete representation or a frame (Mallat, 2009).

Mathematically, if $\mathbf{F}_{n}$ is the orthonormal seislet transform for $n$-th frequency or plane wave, then, for any data vector $\mathbf{d}$,

$$
\sum_{n=1}^{N}\left\|\mathbf{F}_{n} \mathbf{d}\right\|^{2}=\sum_{n=1}^{N} \mathbf{d}^{T} \mathbf{F}_{n}^{T} \mathbf{F}_{n} \mathbf{d}=\sum_{n=1}^{N}\|\mathbf{d}\|^{2}=N\|\mathbf{d}\|^{2}
$$




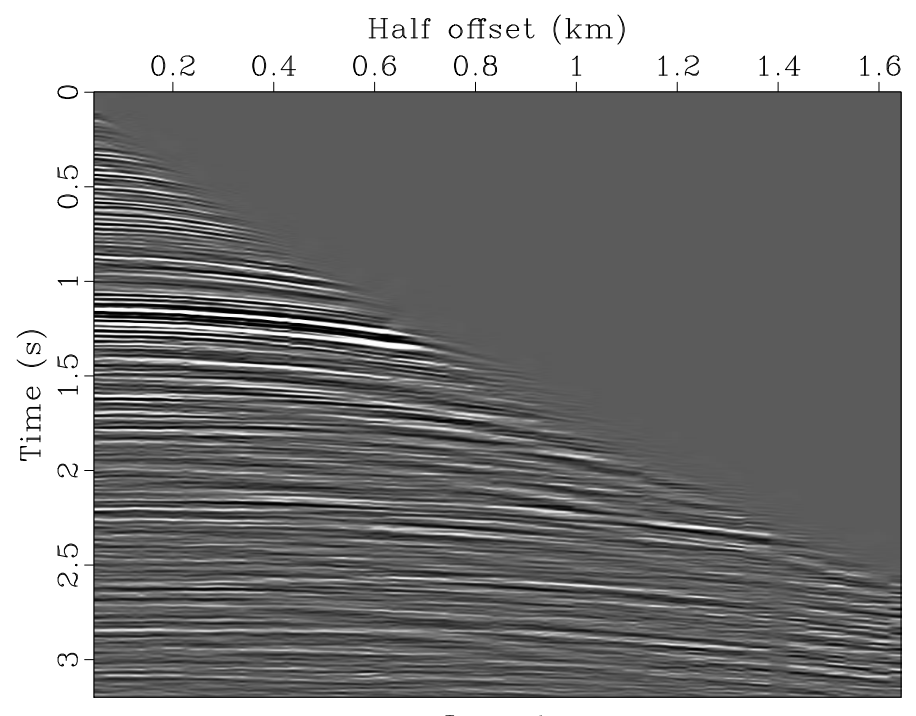

Input

(a)

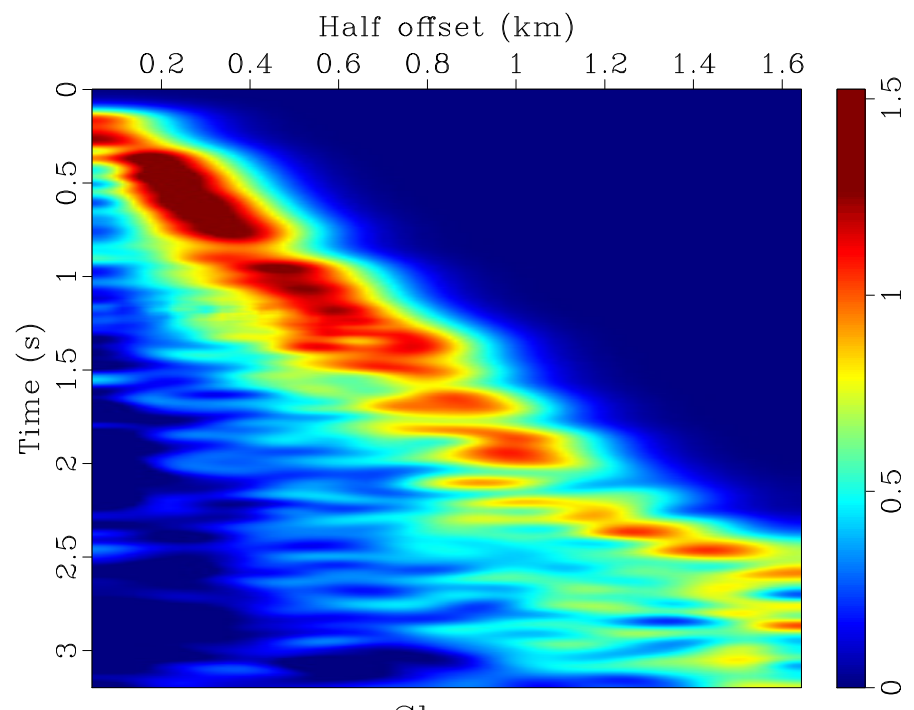

Slope

(b)

Figure 7: Common-midpoint gather (a) and local slopes estimated by plane-wave destruction (b). 


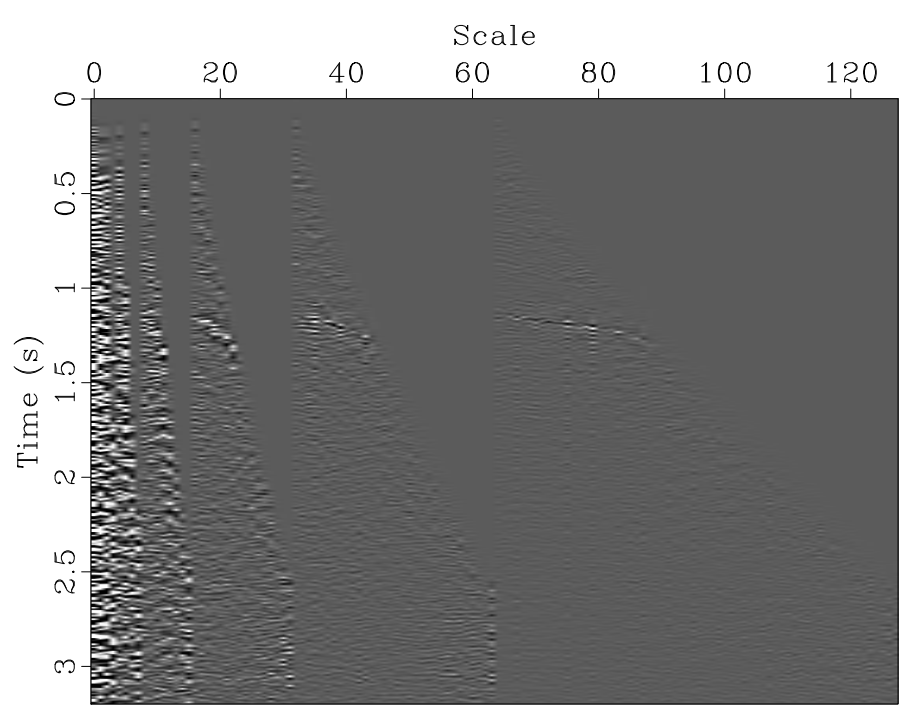

Seislet Transform

(a)

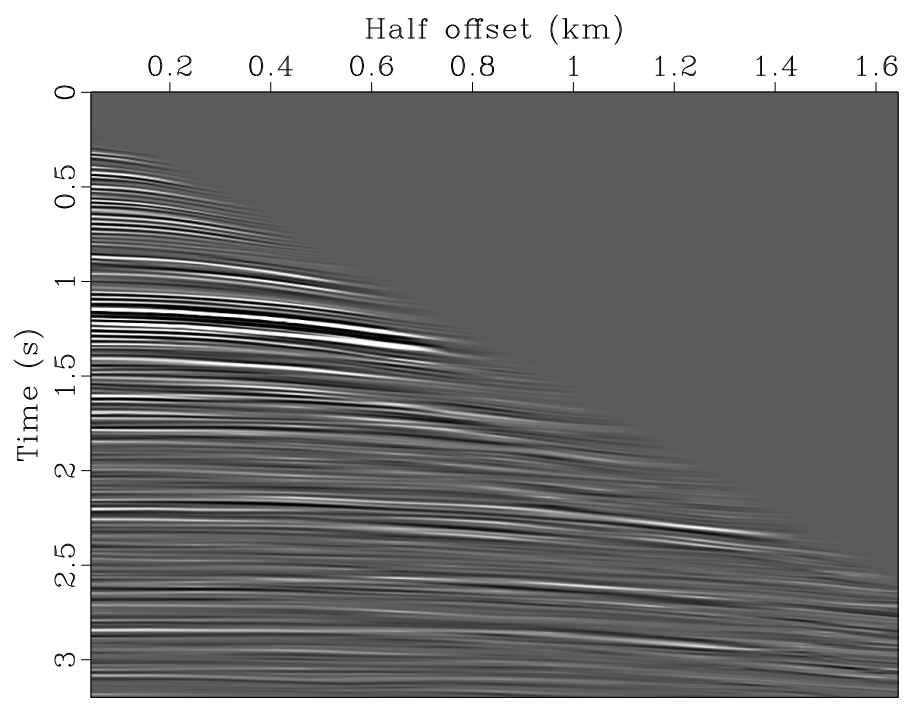

Inverse Seislet Transform (5\%)

(b)

Figure 8: Seislet transform of the input gather (a) and (a) and data reconstruction using only $5 \%$ of significant seislet coefficients (b). 


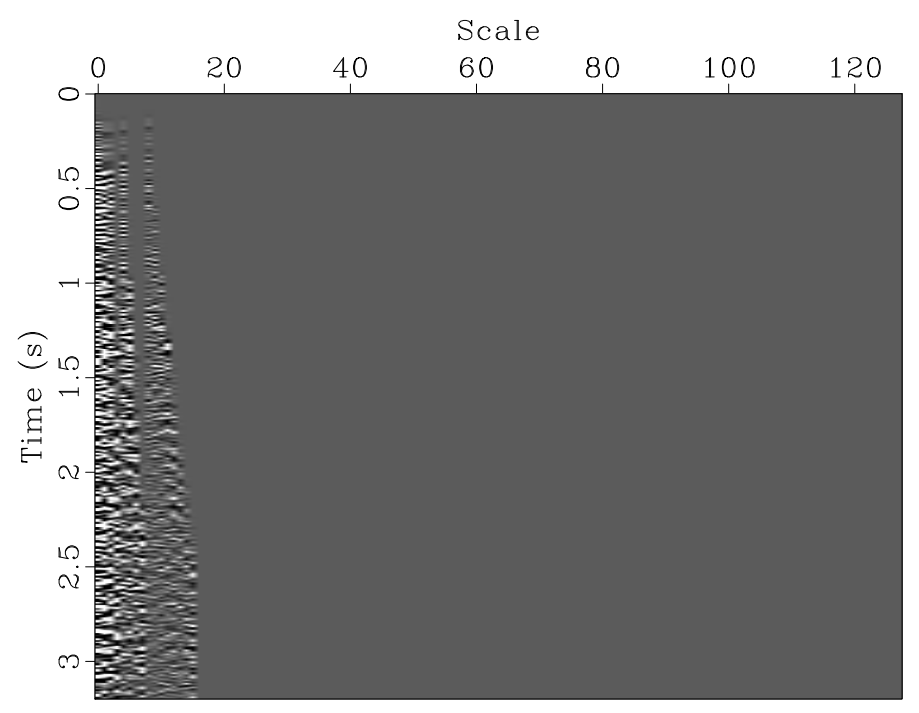

Seislet Transform

(a)

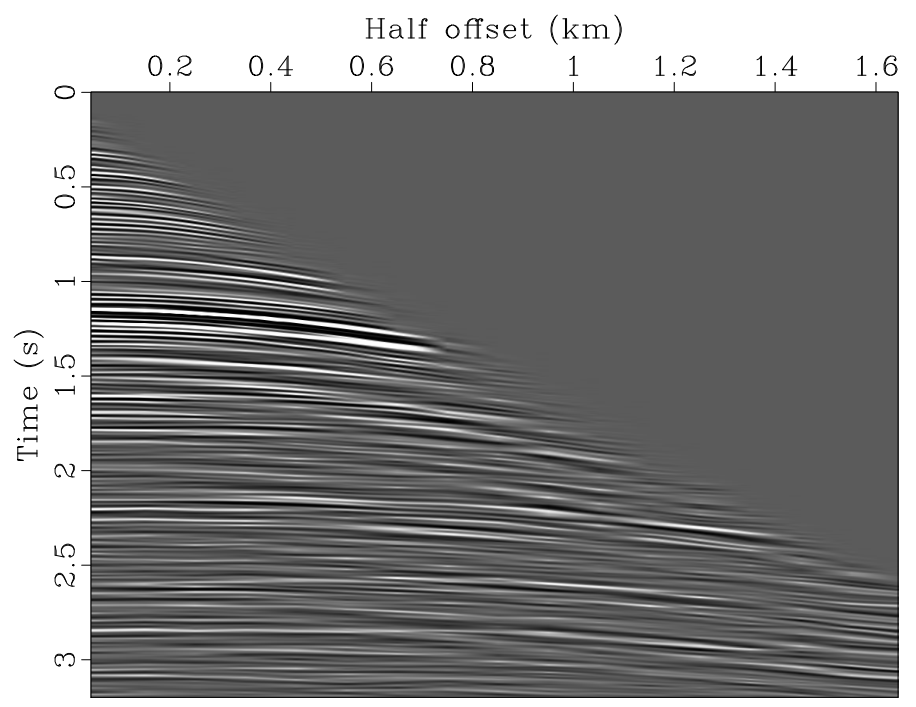

Denoising result

(b)

Figure 9: Zeroing seislet difference coefficients at fine scales (a) enables effective denoising of the reconstructed data (b). 


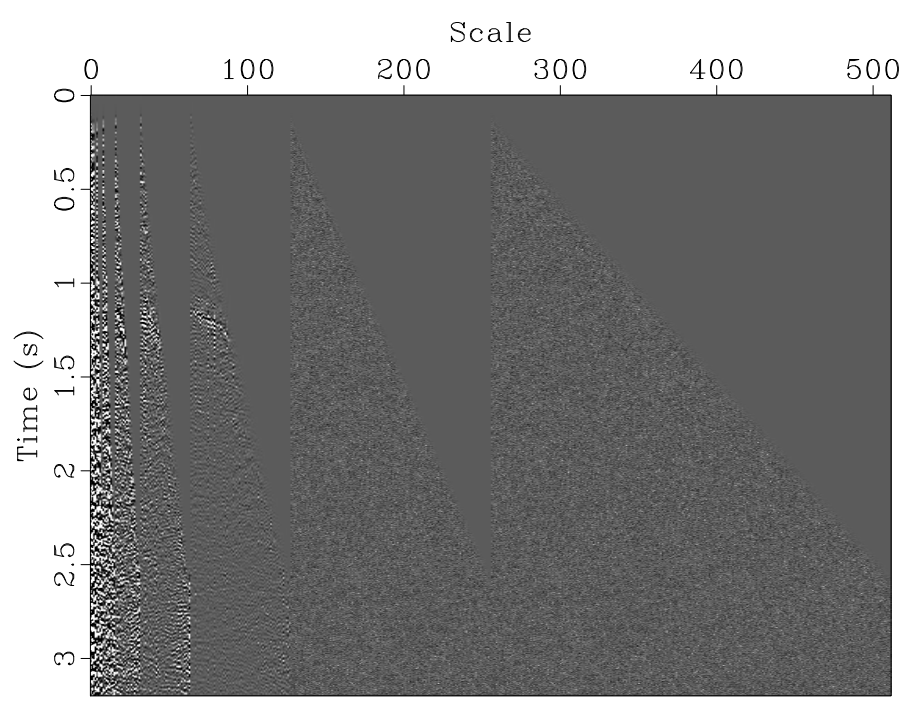

Seislet Transform

(a)

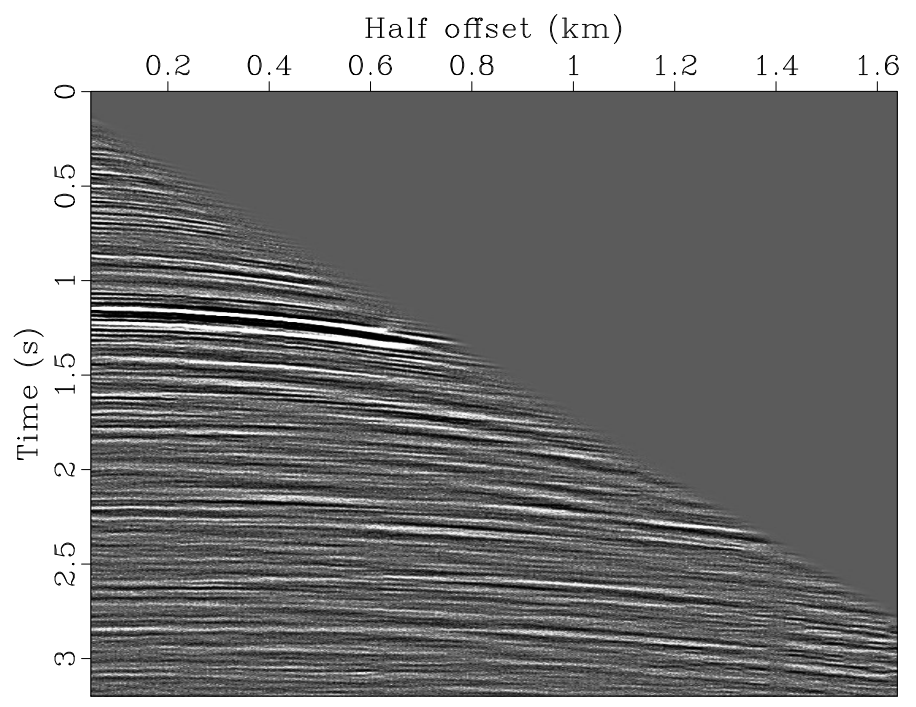

Resampled by 4

(b)

Figure 10: Extending seislet transform with random noise (a) enables trace interpolation in the reconstructed data. The interpolated section (b) has 4 times more traces than the original shown in Figure $7(\mathrm{a})$. 


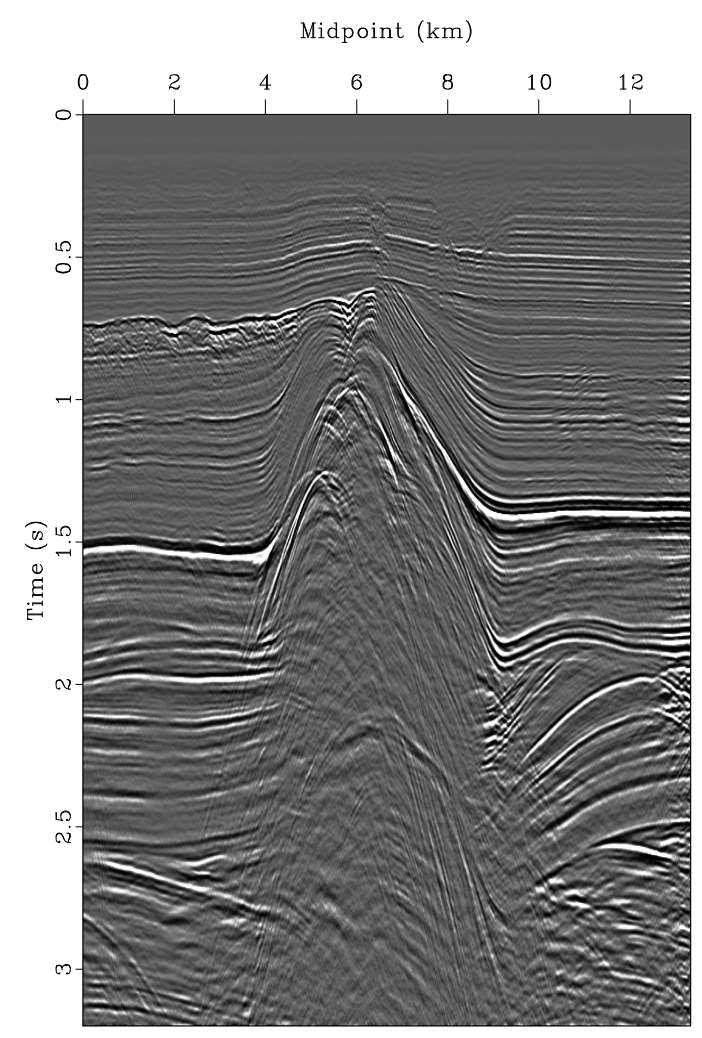

NMO Stack

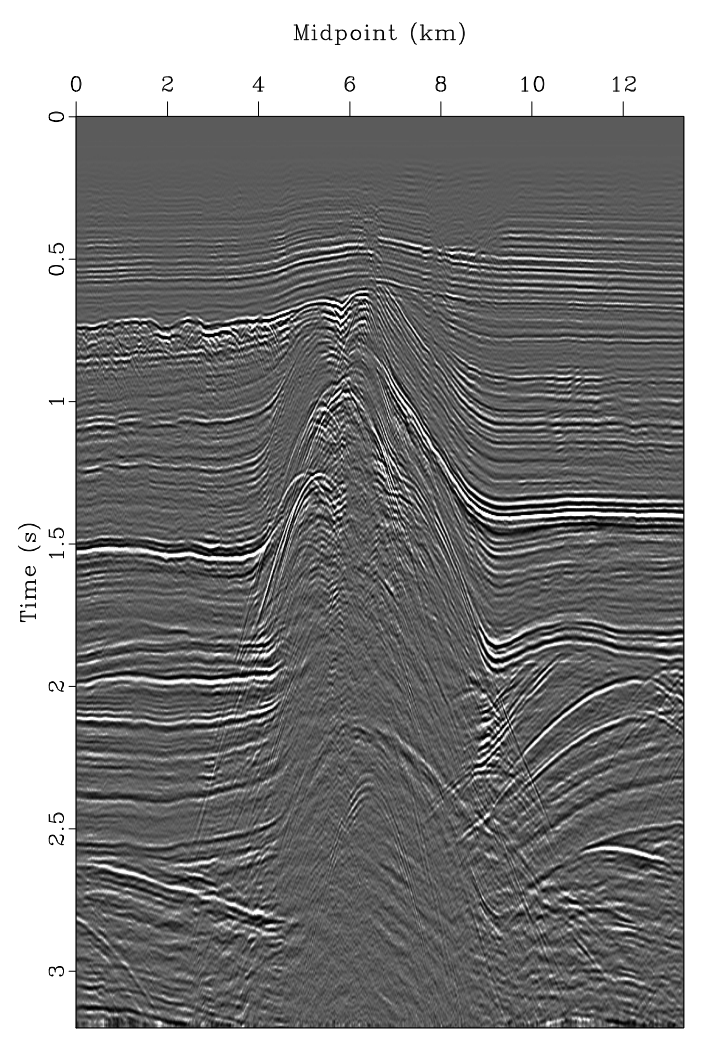

Seislet Stack

Figure 11: Left: conventional normal-moveout stack. Right: seislet stack. 


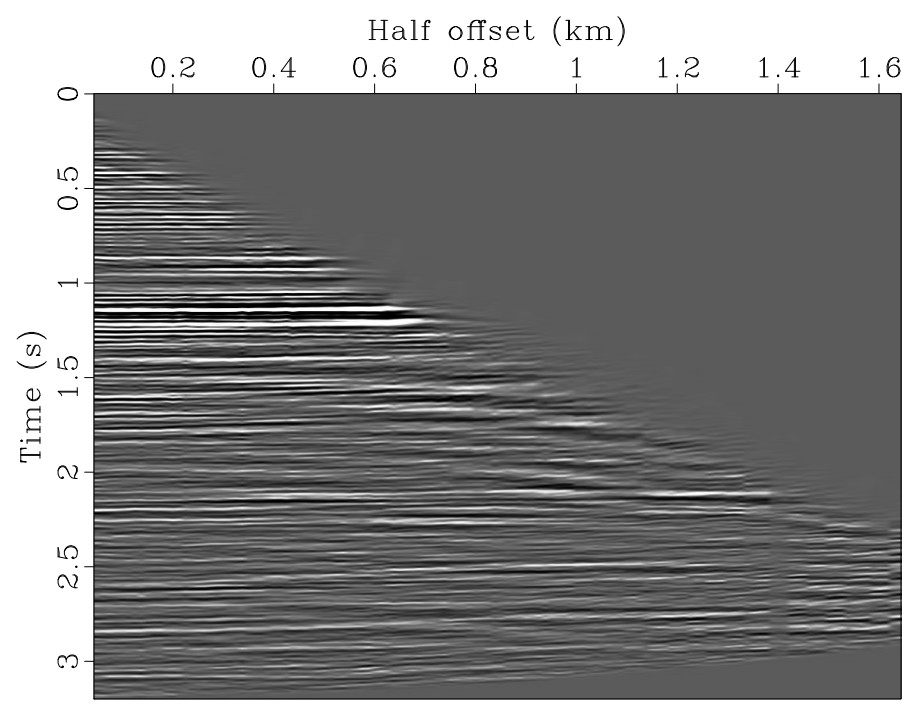

Normal Moveout

(a)

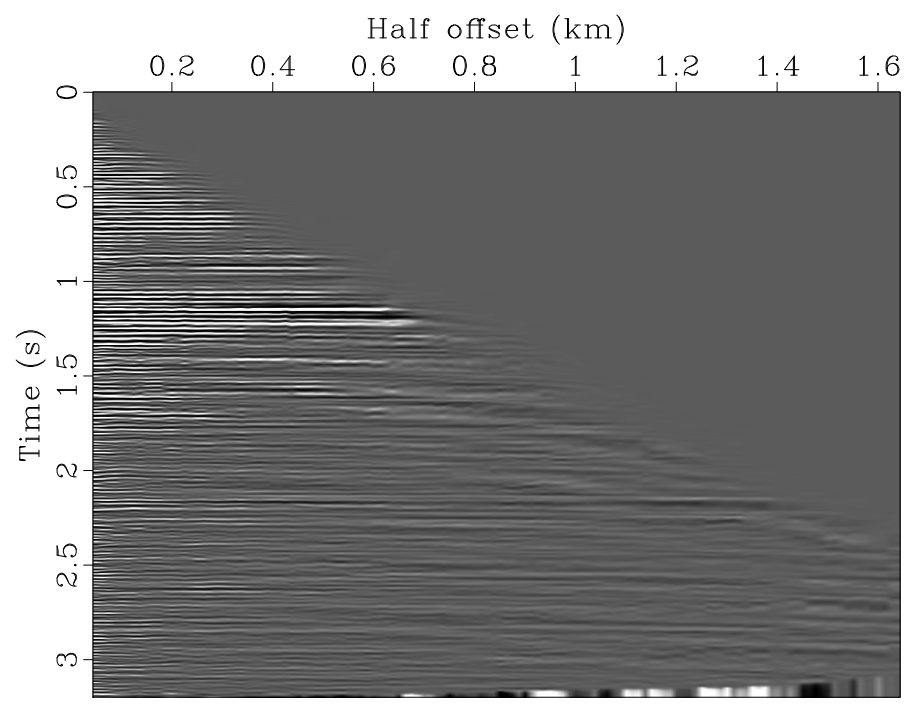

Seislet Moveout

(b)

Figure 12: Input gather after normal moveout correction (a) and effective seislet moveout (b). 
which means that all transforms taken together constitute a tight frame with constant $N$.

For example, in the 1-D case, one can find appropriate frequencies by autoregressive spectral analysis (Burg, 1975; Marple, 1987). We define the algorithm for the 1-D seislet frame as follows:

1. Select a range of coefficients $Z_{1}, Z_{2}, \ldots, Z_{k}$. When using autoregressive spectral analysis, these coefficients are simply the roots of the prediction-error filter. Alternatively, they can be defined from an appropriate range of frequencies $\omega_{1}, \omega_{2}, \ldots, \omega_{k}$.

2. For each of the coefficients, perform the 1-D seislet transform.

Because of its over-completeness, a frame representation for a given signal is not unique. In order to assure that different frequency components do not leak into other parts of the frame, it is advantageous to employ sparseness-promoting inversion. We adopt a nonlinear shaping regularization scheme (Fomel, 2008), analogous to the sparse inversion method of Daubechies et al. (2004), and define sparse decomposition as an iterative process

$$
\begin{aligned}
\widehat{\mathbf{f}}_{k+1} & =\mathbf{S}\left[\mathbf{F} \mathbf{d}+\left(\mathbf{I}-\mathbf{F} \mathbf{F}^{\prime}\right) \widehat{\mathbf{f}}_{k}\right], \\
\mathbf{f}_{k+1} & =\mathbf{f}_{k}+\mathbf{F} \mathbf{d}-\mathbf{F} \mathbf{F}^{\prime} \widehat{\mathbf{f}}_{k+1},
\end{aligned}
$$

where $\mathbf{f}_{k}$ is the seislet frame at $k$-th iteration, $\widehat{\mathbf{f}}_{k}$ is an auxiliary quantity, $\mathbf{d}$ is input data, $\mathbf{I}$ is the identity operator, $\mathbf{F}$ and $\mathbf{F}^{\prime}$ are frame construction and deconstruction operators

$$
\begin{aligned}
\mathbf{F} & \equiv\left[\begin{array}{llll}
\mathbf{F}_{1} & \mathbf{F}_{2} & \cdots & \mathbf{F}_{k}
\end{array}\right]^{T}, \\
\mathbf{F}^{\prime} & \equiv\left[\begin{array}{llll}
\mathbf{F}_{1}^{-1} & \mathbf{F}_{2}^{-1} & \cdots & \mathbf{F}_{k}^{-1}
\end{array}\right],
\end{aligned}
$$

where $\mathbf{F}_{j}$ is the seislet transform for an individual frequency, and $\mathbf{S}$ is a nonlinear shaping operator, such as soft thresholding (Donoho, 1995). The iteration 15-16 starts with $\mathbf{f}_{0}=\mathbf{0}$ and $\widehat{\mathbf{f}}_{0}=\mathbf{F} \mathbf{d}$ and is related to the linearized Bregman iteration (Osher et al., 2005; Yin et al., 2008). We find that a small number of iterations is usually sufficient for convergence and achieving both model sparseness and data recovery.

\section{1-D data analysis with 1-D seislet frame}

We use a simple synthetic test to verify the compression effectiveness of 1 -D seislet frame. A test signal mixing two sinusoids with different frequencies and some random noise is displayed in Figure 13(a). We use a prediction-error filter to detect the signal frequencies and to design the corresponding seislet frame. The result is shown in Figure 13(b). The 1-D seislet frame algorithm with shaping regularization compresses 
the sinusoidal signal into two nearly perfect impulses with some dispersive random noise. For comparison, we also apply DFT and DWT to transform the signal (Figures $13(\mathrm{~d})$ and $13(\mathrm{c})$ ). In the Fourier transform domain, the signal appears as two impulses corresponding to the chosen frequency components. The resolution is not perfect because of spectral leakage caused by non-periodic input data. In the wavelet domain, the transform coefficients are not compressed well. For further comparison, we plot the coefficients in the three different transform domains, sorted from large to small, on a decibel scale (Figure 14). The significantly faster rate of coefficient decay shows the superiority of the 1-D seislet frame in compressing sinusoidal signals.

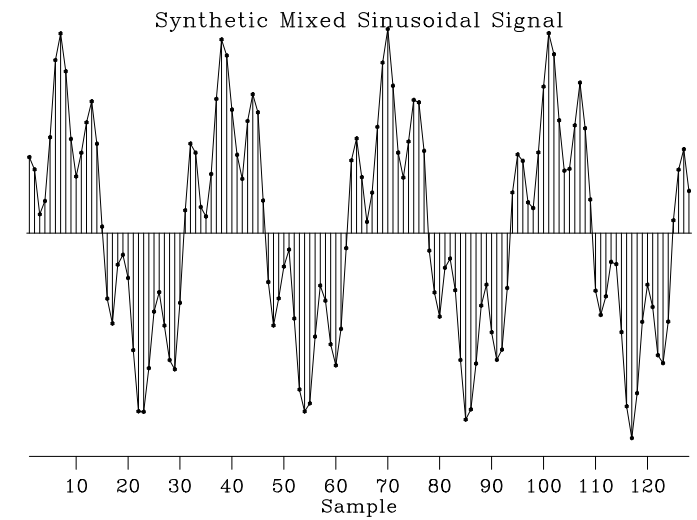

(a)

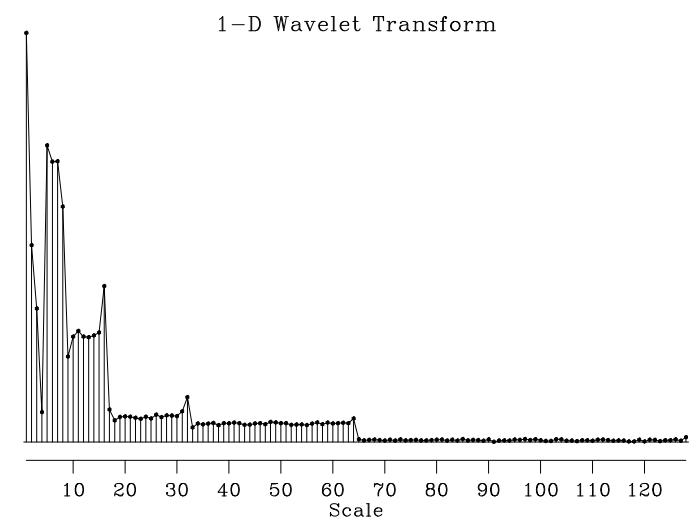

(c)

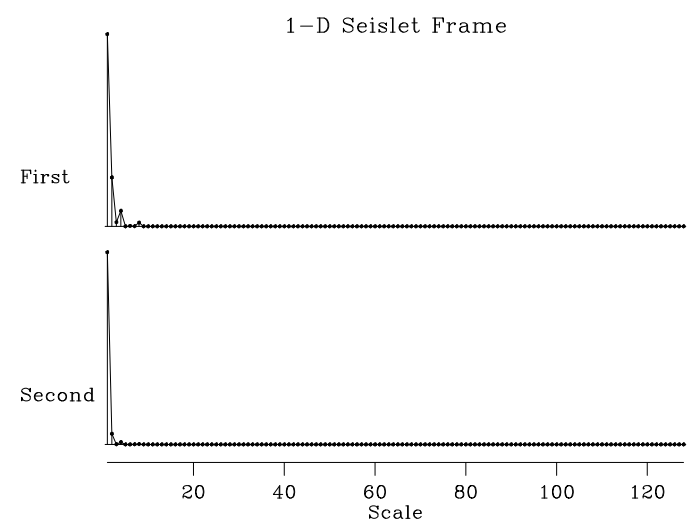

(b)

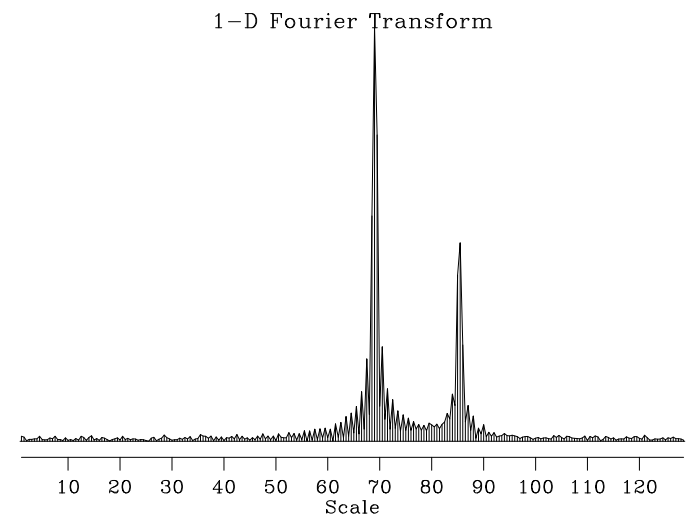

(d)

Figure 13: Mixed sinusoidal signal (a), 1-D seislet frame (b), 1-D wavelet transform (c), and 1-D Fourier transform (d).

\section{2-D data analysis with 1-D seislet frame}

To analyze 2-D data, one can apply 1-D seislet frame in the distance direction after the Fourier transform in time (the $F-X$ domain). In this case, different frame frequencies correspond to different plane-wave slopes (Canales, 1984). We use a simple planewave synthetic model to verify this observation (Figure $15(\mathrm{a})$ ). The $F-X$ plane is 


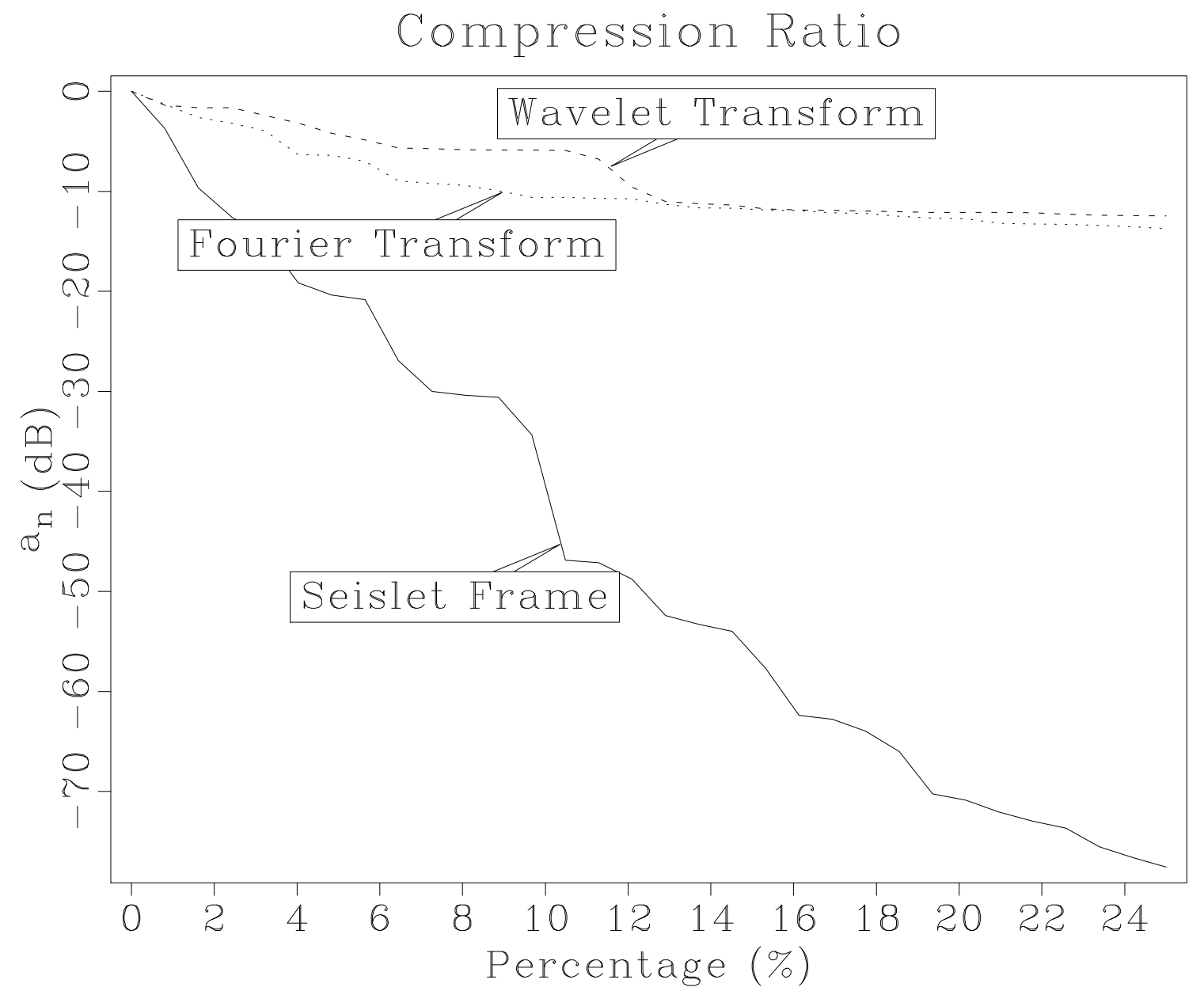

Figure 14: Compression comparison between digital Fourier transform, digital wavelet transform, and 1-D seislet frame. Transform coefficients are sorted from large to small, normalized, and plotted on a decibel scale. 
shown in Figure 15(b). We find a prediction-error-filter (PEF) in each frequency slice and detect its roots to select appropriate spatial frequencies. We use Burg's algorithm for PEF estimation (Burg, 1975; Claerbout, 1976) and an eigenvalue-based algorithm for root finding (Edelman and Murakami, 1995). The seislet coefficients and the corresponding recovered plane-wave components are shown in Figure 16. Similarly to the 1-D example, information from different plane-waves gets strongly compressed in the transform domain.

\section{2-D data analysis with 2-D seislet frame}

To show an example of 2-D data analysis with 2-D seislet frames, we use the CMP gather from Figure 7(a). We try two different choices for selecting a set of dip fields for the frame construction.

First, we define dip fields by scanning different constant dips (Figure 17(a)). In this case, the zero-scale coefficients out of the 2-D seislet frame correspond to the slant-stack (Radon transform) gather (Figure 18(a)). Figure 19(a) shows randomly selected example frame functions for the 2-D seislet frame using constant dips

Our second choice is a set of dip fields defined by the hyperbolic shape of seismic events on the CMP gather:

$$
t(x)=\sqrt{t_{0}^{2}+\frac{x^{2}}{v^{2}}},
$$

where $t(x)$ is traveltime for reflection at offset $x, t_{0}$ is the zero-offset traveltime, and $v$ is the root-mean-square velocity. For a range of constant velocities, the direct relationship between dip and velocity is given by

$$
p=\frac{d t}{d x}=\frac{x}{v^{2} t} .
$$

The dip field $p(x, t, v)$ is shown in Figure 17(b). Analogously to the case of constant dips, the frame coefficients at the zero scale correspond to the hyperbolic Radon transform (Thorson and Claerbout, 1985), with the primary and multiple reflections distributed in different velocity ranges (Figure 18(b)). Figure 19(b) shows randomly selected frame functions for the 2-D seislet frame with varying dip fields defined by a range of constant velocities.

\section{DISCUSSION}

How efficient are the proposed algorithms? The CPU times, in our implementation, are shown in Table 1. They confirm that, while the seislet transform and frame can be more expensive than FFT or DWT, they are still comfortably efficient in practice. In applications of the 2-D seislet transform, the main cost may not be in 


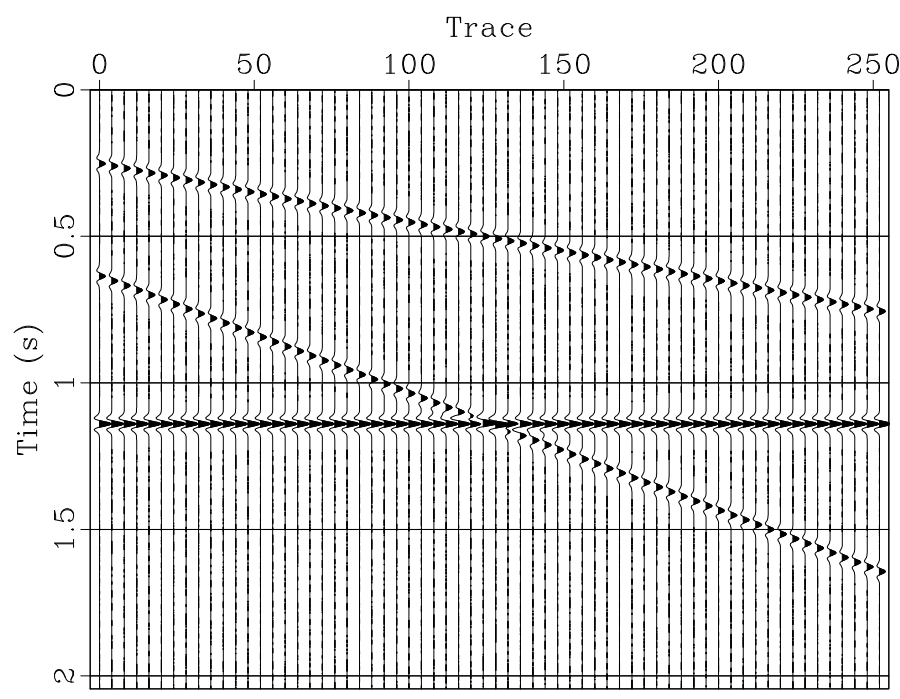

Input

(a)

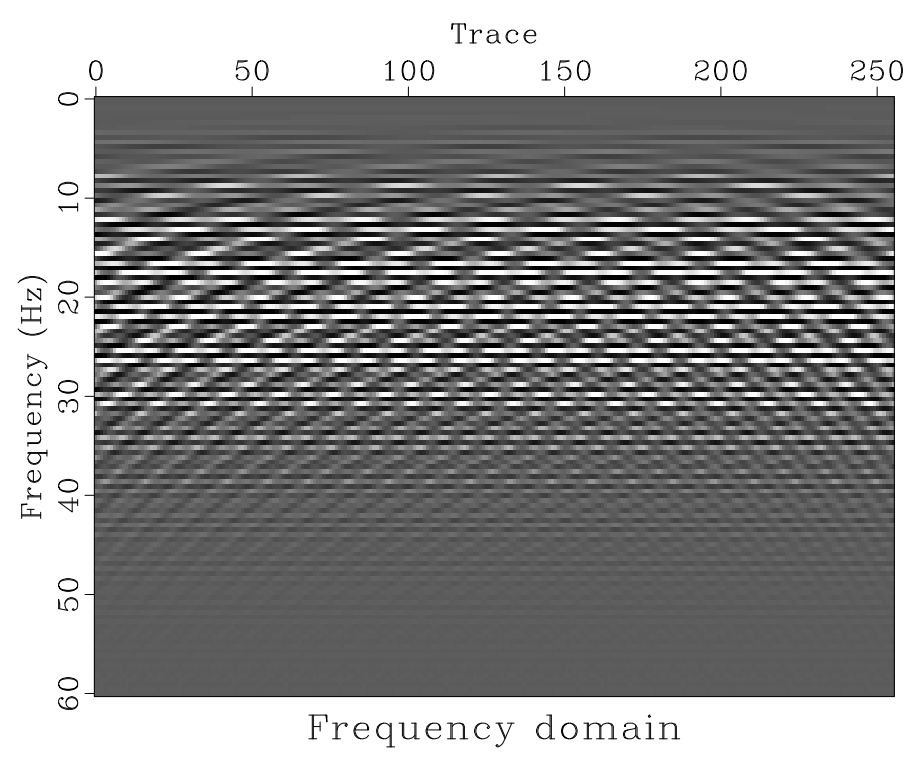

(b)

Figure 15: Synthetic plane-wave data (a) and corresponding Fourier transform along the time direction (b).

\begin{tabular}{|c|c|c|c|c|}
\hline Data size & 1-D FFT & 1-D DWT & 1-D seislet & 2-D seislet \\
\hline $1024 \times 1024$ & 0.06 & 0.03 & 0.17 & 1.03 \\
\hline $512 \times 512$ & 0.02 & 0.01 & 0.04 & 0.22 \\
\hline
\end{tabular}

Table 1: CPU times (in seconds) for different transforms. 

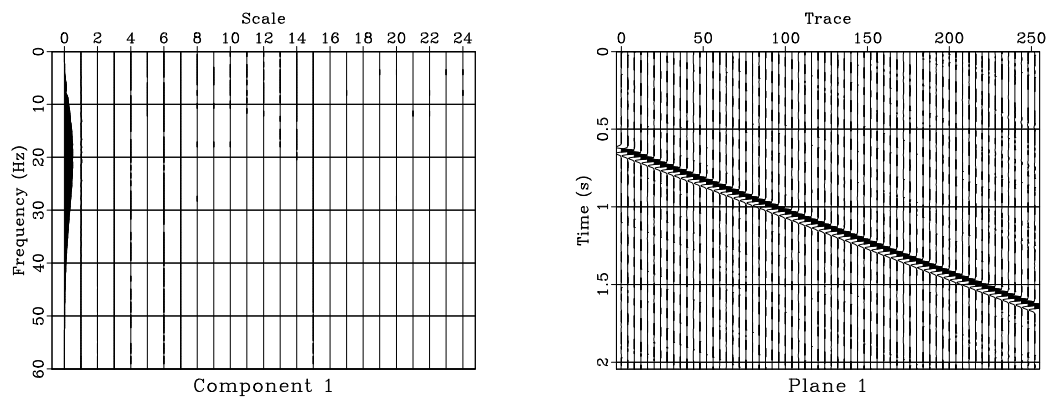

(a)
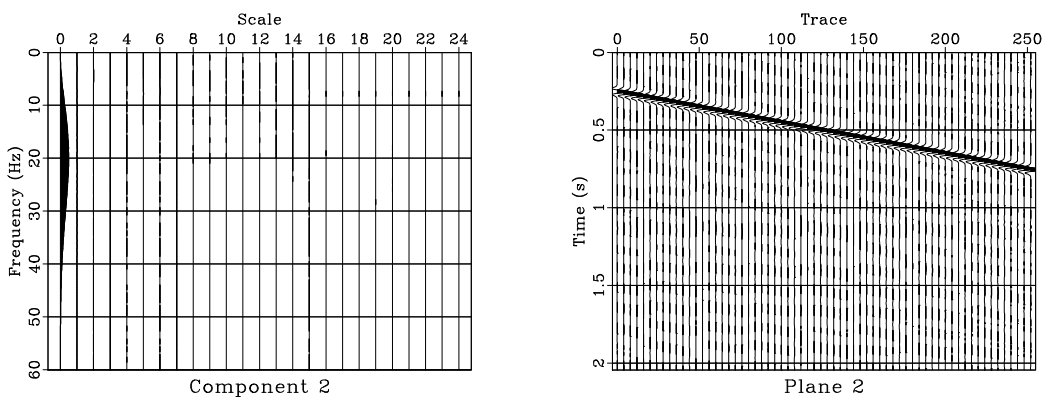

(b)
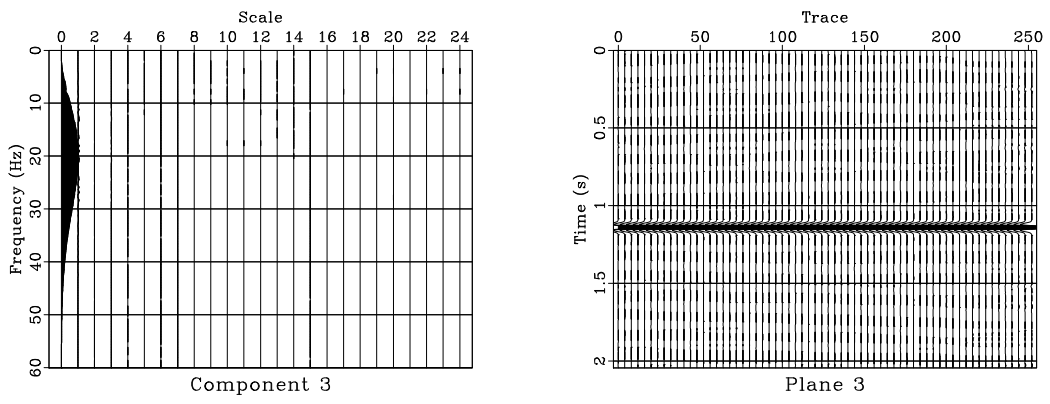

(c)

Figure 16: Seislet coefficients (left) and corresponding recovered plane-wave components (right) for three different parts of the $1-D$ seislet frame in the $F-X$ domain. 


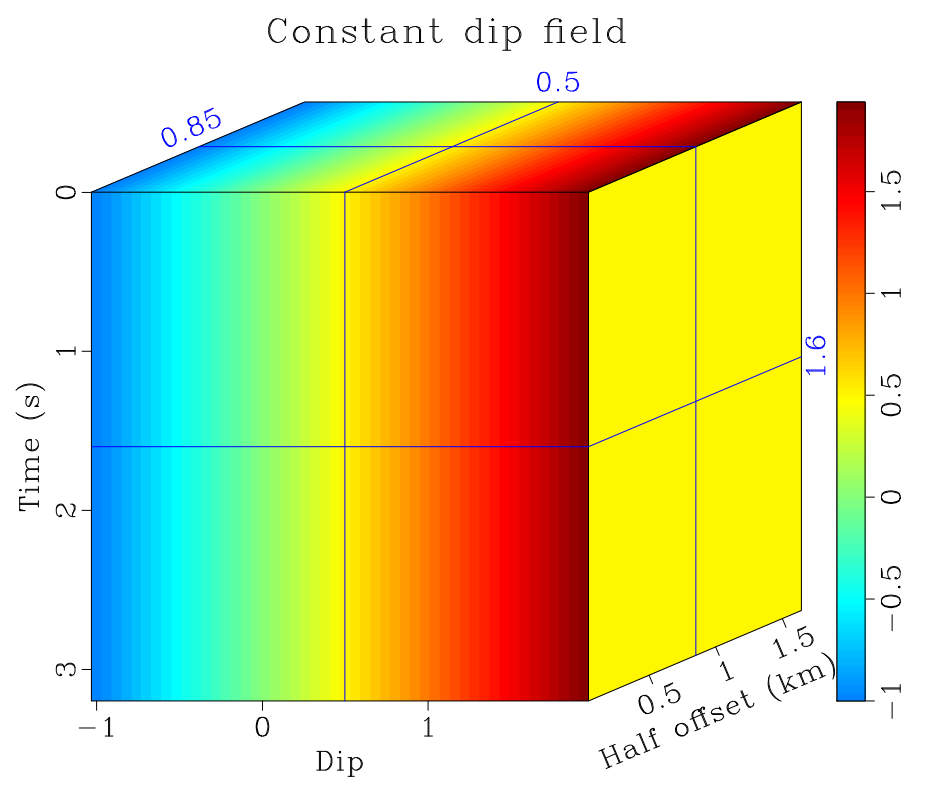

(a)

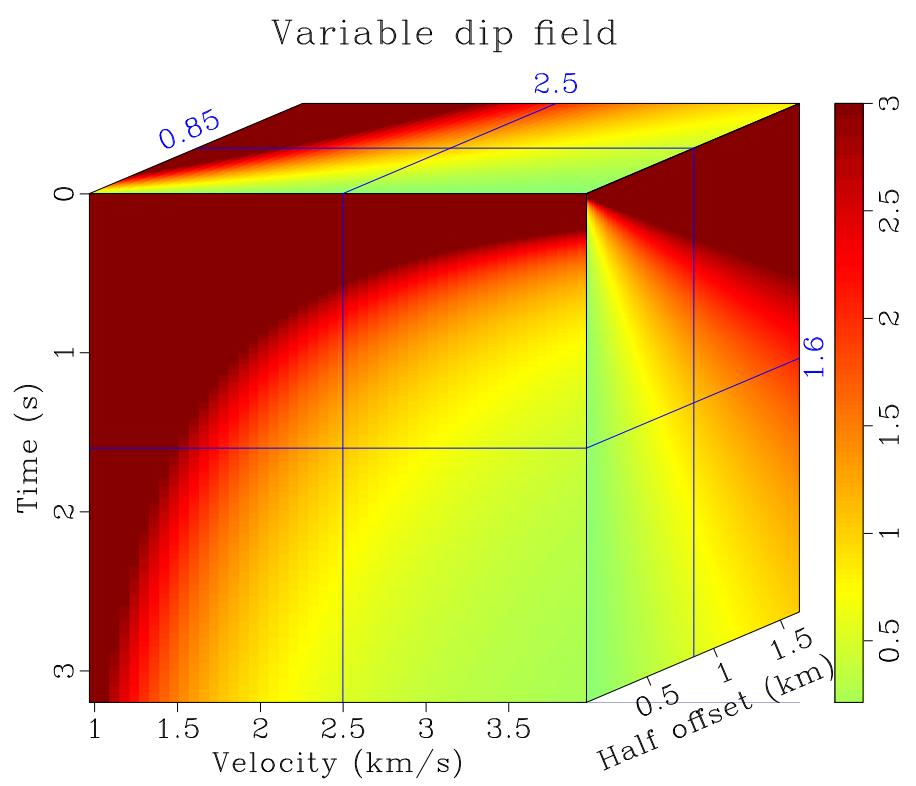

(b)

Figure 17: Constant dip field (a) and time and space varying dip field (b). 


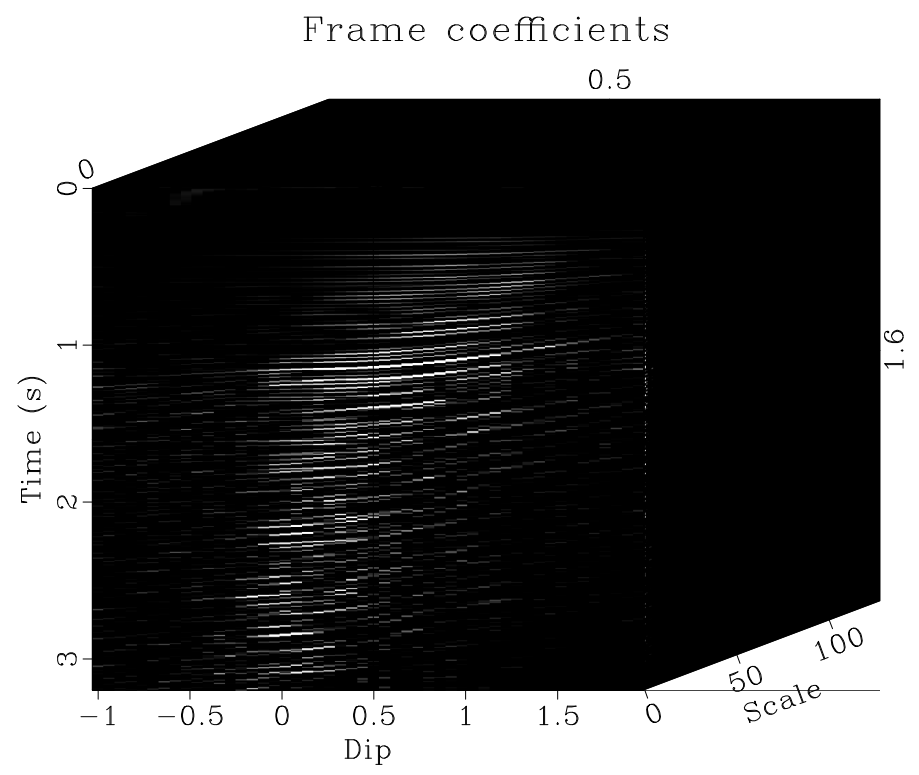

(a)

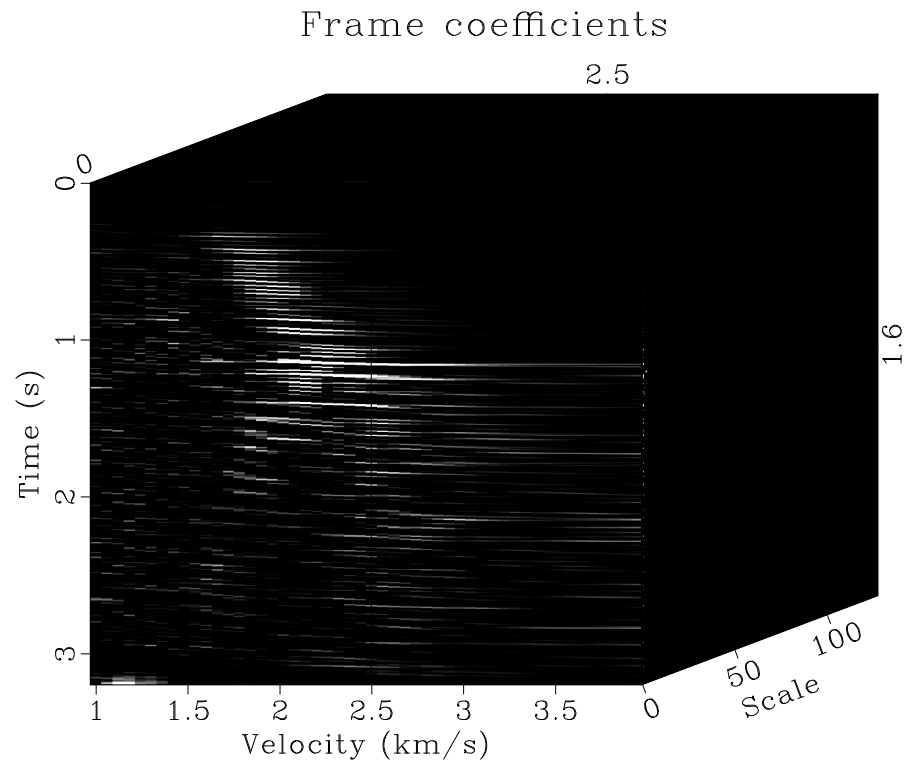

(b)

Figure 18: 2-D seislet frame coefficients with constant dip field (a) and with varying dip field (b). 


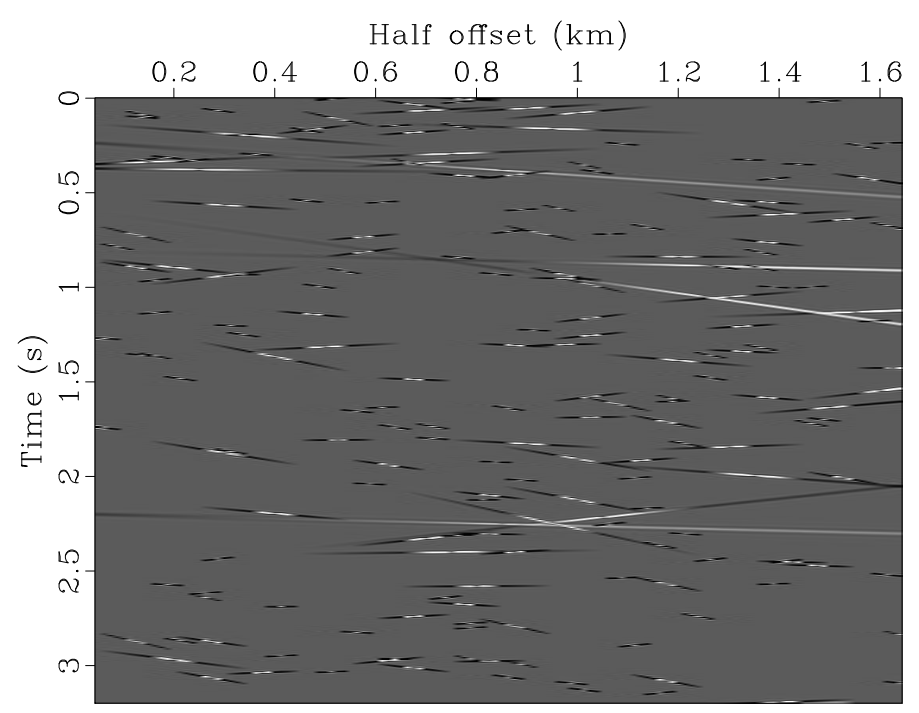

Constant dip field

(a)

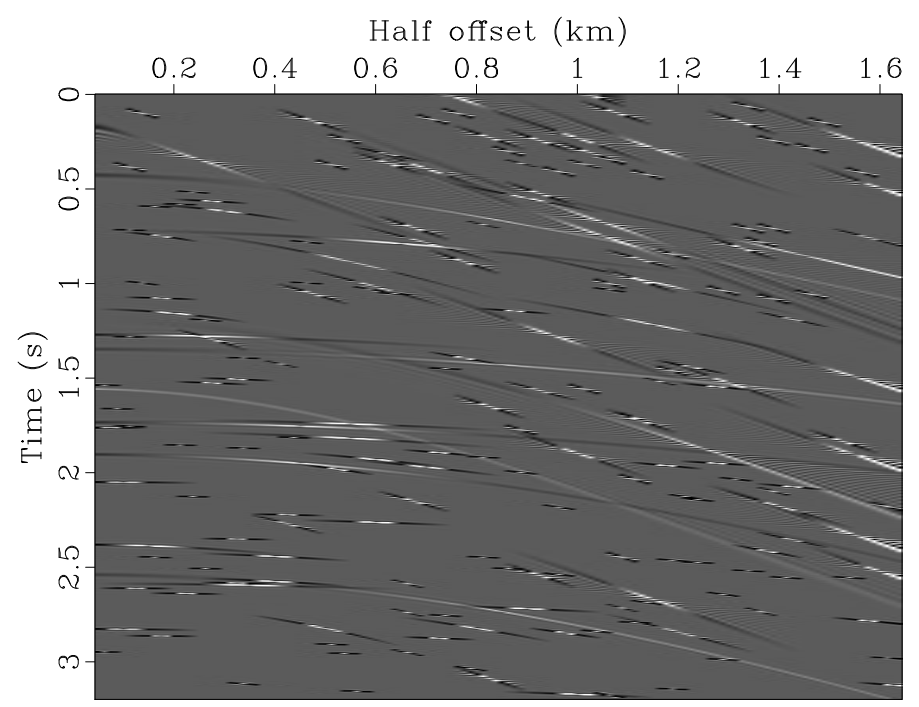

Variable dip field

(b)

Figure 19: Randomly selected representative frame functions for 2-D seislet frame with constant dip field (a) and varying dip field (b). 
the transform itself but in iterative estimation of the slope fields. In practical largescale applications, it is advantageous to break the input data in parts and process them in parallel.

How effective are the seislet transform and frame in compressing seismic data? In the case of the 2-D seislet transform that requires a slope field, it appears that one would need to store this field in addition to the compressed data. However, since we force the estimated slopes to be smooth, the slope field can be easily compressed with one of the classic compression algorithms. Consider the example in Figure 2. Suppose that we apply lossy compression and require $99 \%$ of the energy to be preserved. The seislet transform compression ratio in this case is less that $1 \%$ while the corresponding wavelet transform ratio is $26 \%$. Applied to the smooth slope field from Figure 2(b), the wavelet transform compresses it to about $0.1 \%$. This example shows that compressing seismic data with the seislet transform and the corresponding slope field with the wavelet transform can be significantly more effective that trying to compress seismic data with the wavelet transform.

\section{CONCLUSIONS}

We have introduced a new digital transform named seislet transform because of its ability to characterize and compress seismic data in the manner similar to that of digital wavelet transforms. We define the seislet transform by combining the wavelet lifting scheme with local plane-wave destruction. In 1-D, the seislet transform follows sinusoidal components. In 2-D, it follows locally plane events. When more than one sinusoid or more than one local slope are applied for the analysis, the transform turns into an overcomplete representation or a frame. The seislet transform and seislet frame can achieve a better compression ratio than either the digital Fourier transform (DFT) or the digital wavelet transform (DWT).

The seislet transform provides a convenient orthogonal basis with the basis functions spanning different scales analogously to those of the digital wavelet transform but aligned along the dominant seismic events. Traditional signal analysis operations such as denoising and trace interpolation become simply defined in the seislet domain and allow for efficient algorithms. Seismic stacking also has a simple meaning of the zeroth-order seislet coefficient computed in an optimally efficient manner by recursive partial stacking and thus avoiding the usual problems with wavelet stretch and nonhyperbolic moveouts.

\section{ACKNOWLEDGMENTS}

We thank BGP Americas for a partial financial support of this work. The first author is grateful to Huub Douma for inspiring discussions and for suggesting the name "seislet". This publication is authorized by the Director, Bureau of Economic Geology, The University of Texas at Austin. 


\section{APPENDIX A}

\section{REVIEW OF PLANE-WAVE DESTRUCTION}

This appendix reviews the basic theory of plane-wave destruction (Fomel, 2002).

Following the physical model of local plane waves, we define the mathematical basis of plane-wave destruction filters via the local plane differential equation (Claerbout, 1992)

$$
\frac{\partial P}{\partial x}+\sigma \frac{\partial P}{\partial t}=0,
$$

where $P(t, x)$ is the wave field, and $\sigma$ is the local slope, which may also depend on $t$ and $x$. In the case of a constant slope, equation A-1 has the simple general solution

$$
P(t, x)=f(t-\sigma x),
$$

where $f(t)$ is an arbitrary waveform. Equation A-2 is nothing more than a mathematical description of a plane wave.

If we assume that the slope $\sigma$ does not depend on $t$, we can transform equation A-1 to the frequency domain, where it takes the form of the ordinary differential equation

$$
\frac{d \hat{P}}{d x}+i \omega \sigma \hat{P}=0
$$

and has the general solution

$$
\hat{P}(x)=\hat{P}(0) e^{i \omega \sigma x},
$$

where $\hat{P}$ is the Fourier transform of $P$. The complex exponential term in equation A-4 simply represents a shift of a $t$-trace according to the slope $\sigma$ and the trace separation $x$.

In the frequency domain, the operator for transforming the trace $x-1$ to the neighboring trace $x$ is a multiplication by $e^{i \omega \sigma}$. In other words, a plane wave can be perfectly predicted by a two-term prediction-error filter in the $F$ - $X$ domain:

$$
a_{0} \hat{P}(x)+a_{1} \hat{P}(x-1)=0,
$$

where $a_{0}=1$ and $a_{1}=-e^{i \omega \sigma}$. The goal of predicting several plane waves can be accomplished by cascading several two-term filters. In fact, any $F-X$ prediction-error filter represented in the $Z$-transform notation as

$$
A\left(Z_{x}\right)=1+a_{1} Z_{x}+a_{2} Z_{x}^{2}+\cdots+a_{N} Z_{x}^{N}
$$

can be factored into a product of two-term filters:

$$
A\left(Z_{x}\right)=\left(1-\frac{Z_{x}}{Z_{1}}\right)\left(1-\frac{Z_{x}}{Z_{2}}\right) \cdots\left(1-\frac{Z_{x}}{Z_{N}}\right),
$$


where $Z_{1}, Z_{2}, \ldots, Z_{N}$ are the zeroes of polynomial A-6. According to equation A-5, the phase of each zero corresponds to the slope of a local plane wave multiplied by the frequency. Zeroes that are not on the unit circle carry an additional amplitude gain not included in equation A-3.

In order to incorporate time-varying slopes, we need to return to the time domain and look for an appropriate analog of the phase-shift operator A-4 and the planeprediction filter A-5. An important property of plane-wave propagation across different traces is that the total energy of the propagating wave stays invariant throughout the process: the energy of the wave at one trace is completely transmitted to the next trace. This property is assured in the frequency-domain solution A-4 by the fact that the spectrum of the complex exponential $e^{i \omega \sigma}$ is equal to one. In the time domain, we can reach an equivalent effect by using an all-pass digital filter. In the $Z$-transform notation, convolution with an all-pass filter takes the form

$$
\hat{P}_{x+1}\left(Z_{t}\right)=\hat{P}_{x}\left(Z_{t}\right) \frac{B\left(Z_{t}\right)}{B\left(1 / Z_{t}\right)},
$$

where $\hat{P}_{x}\left(Z_{t}\right)$ denotes the $Z$-transform of the corresponding trace, and the ratio $B\left(Z_{t}\right) / B\left(1 / Z_{t}\right)$ is an all-pass digital filter approximating the time-shift operator $e^{i \omega \sigma}$. In finite-difference terms, equation A-8 represents an implicit finite-difference scheme for solving equation A-1 with the initial conditions at a constant $x$. The coefficients of filter $B\left(Z_{t}\right)$ can be determined, for example, by fitting the filter frequency response at low frequencies to the response of the phase-shift operator. This leads to a version of Thiran's maximally-flat all-pass fractional-delay filters (Thiran, 1971; Välimäki and Laakso, 2001).

Taking both dimensions into consideration, equation A-8 transforms to the prediction equation analogous to $\mathrm{A}-5$ with the 2 -D prediction filter

$$
A\left(Z_{t}, Z_{x}\right)=1-Z_{x} \frac{B\left(Z_{t}\right)}{B\left(1 / Z_{t}\right)} .
$$

In order to characterize several plane waves, we can cascade several filters of the form A-9 in a manner similar to that of equation A-7. A modified version of the filter $A\left(Z_{t}, Z_{x}\right)$, namely the filter

$$
C\left(Z_{t}, Z_{x}\right)=A\left(Z_{t}, Z_{x}\right) B\left(1 / Z_{t}\right)=B\left(1 / Z_{t}\right)-Z_{x} B\left(Z_{t}\right),
$$

avoids the need for polynomial division. In case of a 3-point filter $B\left(Z_{t}\right)$, the 2-D filter A-10 has exactly six coefficients. It consists of two columns, each column having three coefficients and the second column being a reversed copy of the first one.

\section{REFERENCES}

Abma, R., and N. Kabir, 2006, 3D interpolation of irregular data with a POCS algorithm: Geophysics, 71, E91-E97. 
Burg, J. P., 1975, Maximum entropy spectral analysis: PhD thesis, Stanford University.

Canales, L. L., 1984, Random noise reduction: 54th Annual International Meeting, SEG, Expanded Abstracts, Session: S10.1.

Candés, E. J., and L. Demanet, 2005, The curvelet representation of wave propagators is optimally sparse: Communications on Pure and Applied Mathematics, 58, 14721528.

Chauris, H., and T. Nguyen, 2008, Seismic demigration/migration in the curvelet domain: Geophysics, S35-S46.

Claerbout, J. F., 1976, Fundamentals of geophysical data processing: Blackwell Scientific Publications.

— 1992 , Earth Soundings Analysis: Processing Versus Inversion: Blackwell Scientific Publications.

— 2008, Basic Earth imaging: Stanford Exploration Project, http://sepwww.stanford.edu/sep/prof/.

Cohen, A., I. Daubechies, and J. Feauveau, 1992, Biorthogonal bases of compactly supported wavelets: Communications on Pure and Applied Mathematics, 45, 485560.

Daubechies, I., M. Defrise, and C. D. Mol, 2004, An iterative thresholding algorithm for linear inverse problems with a sparsity constraint: Communications on Pure and Applied Mathematics, LVII, 1413-1457.

Dessing, F. J., 1997, A wavelet transform approach to seismic processing: PhD thesis, Delft University of Technology.

Do, M. N., and M. Vetterli, 2005, The contourlet transform: an efficient directional multiresolution image representation: IEEE Transactions on Image Processing, 14, 2091-2106.

Donoho, D. L., 1995, De-noising by soft-thresholding: IEEE Trans. on Inform. Theory, 41, 613-627.

Douma, H., and M. V. de Hoop, 2007, Leading-order seismic imaging using curvelets: Geophysics, 72, S231-S248.

Edelman, A., and H. Murakami, 1995, Polynomial roots from companion matrix eigenvalues: Mathematics of Computation, 64, 763-776.

Fomel, S., 2002, Applications of plane-wave destruction filters: Geophysics, 67, 19461960.

— 2006, Towards the seislet transform, in 76th Ann. Internat. Mtg: Soc. of Expl. Geophys., 2847-2850.

—, 2008 , Nonlinear shaping regularization in geophysical inverse problems: 78th Annual International Meeting, SEG, Expanded Abstracts, 2046-2051.

Fomel, S., and V. Grechka, 2001, Nonhyperbolic reflection moveout of P waves. An overview and comparison of reasons: Technical Report CWP-372, Colorado School of Mines.

Foster, D. J., C. C. Mosher, and S. Hassanzadeh, 1994, Wavelet transform methods for geophysical applications: 64th Ann. Internat. Mtg, Soc. of Expl. Geophys., $1465-1468$.

Guo, K., and D. Labate, 2007, Optimally sparse multidimensional representation 
using shearlets: SIAM Journal on Mathematical Analysis, 39, 298-318.

Haldorsen, J. B. U., and P. A. Farmer, 1989, Resolution and NMO-stretch: Imaging by stacking: Geophysical Prospecting, 37, 479-492.

Herrmann, F. J., and G. Hennenfent, 2008, Non-parametric seismic data recovery with curvelet frames: Geophysical Journal International, 173, 233-248.

Herrmann, F. J., D. Wang, G. Hennenfent, and P. Moghaddam, 2007, Curvelet-based seismic data processing: a multiscale and nonlinear approach: Geophysics, 73, A1A5.

Jensen, A., and A. la Cour-Harbo, 2001, Ripples in mathematics: the discrete wavelet transform: Springer.

Kazemeini, S. H., C. Juhlin, K. Zinck-Jorgensen, and B. Norden, 2009, Application of the continuous wavelet transform on seismic data for mapping of channel deposits and gas detection at the $\mathrm{CO} 2$ site, Ketzin, Germany: Geophysical Prospecting, 57, $111-123$.

Liu, Y., and S. Fomel, 2009, Oc-seislet: seislet transform construction with differential offset continuation, in 79th Ann. Internat. Mtg: Soc. of Expl. Geophys., 3228-3232.

Mallat, S., 2009, A wavelet tour of signal processing: The sparse way: Academic Press.

Marple, S. L., 1987, Digital spectral analysis with applications: Prentice Hall.

Morlet, J., 1981, Sampling theory and wave propagation: 51st Ann. Internat. Mtg, Soc. of Expl. Geophys., Session:S15.1.

Osher, S., M. Burger, D. Goldfarb, J. Xu, and W. Yin, 2005, An iterative regularization method for total variation-based image restoration: Multiscale Modeling \& Simulation, 4, 460-489.

Pennec, E. L., and S. Mallat, 2005, Sparse geometrical image representation with bandelets: IEEE Transactions on Image Processing, 14, 423-438.

Starck, J. L., E. J. Candés, and D. L. Donoho, 2002, The curvelet transform for image denoising: IEEE Transactions on Image Processing, 11, 670-684.

Sweldens, W., 1995, The lifting scheme: A new philosophy in biorthogonal wavelet constructions: Wavelet Applications in Signal and Image Processing III, Proc. SPIE 2569, 68-79.

Sweldens, W., and P. Schröder, 1996, Building your own wavelets at home, in Wavelets in Computer Graphics: ACM SIGGRAPH Course notes, 15-87.

Thiran, J.-P., 1971, Recursive digital filters with maximally flat group delay: IEEE Transactions on Circuit Theory, 18, 659-664.

Thorson, J. R., and J. F. Claerbout, 1985, Velocity stack and slant stochastic inversion: Geophysics, 50, 2727-2741.

Välimäki, V., and T. T. Laakso, 2001, Fractional delay filters - design and applications, in Nonuniform sampling: theory and practice: Kluwer Academic/Plenum Publishers, 835-895.

Velisavljevic, V., 2005, Directionlets: anisotropic multi-directional representation with separable filtering: PhD thesis, Ecole Polytechnique Fédérale de Lausanne.

Wapenaar, K., R. Ghose, G. Toxopeus, and J. Fokkema, 2005, The wavelet transform as a tool for geophysical data integration: Integrated Computer-Aided Engineering, $12,5-23$. 
Welland, G., ed., 2003, Beyond wavelets: Academic Press.

Xu, S., Y. Zhang, D. Pham, and G. Lambaré, 2005, Antileakage Fourier transform for seismic data regularization: Geophysics, 70, V87-V95.

Yin, W., S. Osher, D. Goldfarb, and J. Darbon, 2008, Bregman interative algorithoms for $l_{1}$-minimization with application to compressed sensing: SIAM J. Imaging Sciences, 1, 143-168.

Zwartjes, P., and A. Gisolf, 2006, Fourier reconstruction of marine-streamer data in four spatial coordinates: 71, V171-V186.

— 2007 , Fourier reconstruction with sparse inversion: Geophysical Prospecting, 55, 199-221.

Zwartjes, P., and M. Sacchi, 2007, Fourier reconstruction of nonuniformly sampled, aliased seismic data: 72, V21-V32. 